\title{
Incremental elastoplastic analysis for active macro-zones
}

\author{
L. Zito, F. Cucco, E. Parlavecchio and T. Panzeca*,† \\ D.to di Ingegneria Strutturale, Aerospaziale e Geotecnica, Università di Palermo, Viale delle Scienze, \\ 90128 Palermo, Italy
}

\begin{abstract}
SUMMARY
In this paper a strategy to perform incremental elastoplastic analysis using the symmetric Galerkin boundary element method for multidomain type problems is shown. The discretization of the body is performed through substructures, distinguishing the bem-elements characterizing the so-called active macro-zones, where the plastic consistency condition may be violated, and the macro-elements having elastic behaviour only. Incremental analysis uses the well-known concept of self-equilibrium stress field here shown in a discrete form through the introduction of the influence matrix (self-stress matrix). The nonlinear analysis does not use updating of the elastic response inside each plastic loop, but at the end of the load increment only. This is possible by using the self-stress matrix, both, in the predictor phase, for computing the stress caused by the stored plastic strains, and, in the corrector phase, for solving a nonlinear global system, which provides the elastoplastic solution of the active macro-zones. The use of active macro-zones gives rise to a nonlocal and path-independent approach, which is characterized by a notable reduction of the number of plastic iterations. The proposed strategy shows several computational advantages as shown by the results of some numerical tests, reported at the end of this paper. These tests were performed using the Karnak.sGbem code, in which the present procedure was introduced as an additional module. Copyright (C) 2012 John Wiley \& Sons, Ltd.
\end{abstract}

Received 18 October 2010; Revised 21 November 2011; Accepted 9 January 2012

KEY WORDS: multidomain SGBEM; self-equilibrium stress equation; elastoplastic analysis; active macro-zones

\section{INTRODUCTION}

The symmetric Galerkin boundary element method (SGBEM) has been utilized in elastoplastic structural analysis since the eighties. Indeed, this method is perfectly adaptable to nonlinear problems both for the simplicity of the boundary and domain discretization and for the employment of symmetric and sign-defined operators.

Maier and Polizzotto [1] developed a symmetric approach in the boundary element elastoplastic analysis by enforcing, in a Galerkin weighted-residual sense, boundary integral equations and plastic constitutive laws in the cells. In $[2,3]$, an approach to energy formulation for elastic and elastoplastic problems showed how, within the BEM application, the use of piecewise linearized yielding laws for the cell system leads to well-known methods of mathematical programming, also utilized within the finite element method. Maier et al. [4] developed and discussed a symmetric boundary integral equation (BIE) formulation for nonlocal, gradient-dependent plasticity on the basis of its variationally consistent discretization. In [5], a dynamic elastoplastic analysis using elastostatic kernels (i.e. time-independent Green functions for unbounded spaces) was shown. Polizzotto and Zito [6] used the plasticity theory through the backward-difference method inside the

\footnotetext{
*Correspondence to: Teotista Panzeca, Dipartimento di Ingegneria Strutturale e Geotecnica, Universita degli Studi di Palermo, Viale delle Scienze, 90128 Palermo, Italy.

†E-mail: tpanzeca@tiscali.it 
BIEM, showing two variational principles to characterize the solution of step problems. Liu et al. [7] proposed a two-stage interpolation method called quasi-high order element method to solve elastoplastic problems. In [8], the Galerkin-type BEM was used to reformulate the static and kinematic shakedown theorems. Recently, Zhang et al. $[9,10]$ have shown an accurate solution procedure, using the SGBEM and the Complex method, to solve both limit and shakedown analysis problems. Through this strategy the limit or shakedown multiplier is obtained directly as the solution of a mathematical programming problem. This strategy shows the advantage of providing the multiplier with very low CPU times, in comparison with the incremental elastoplastic analysis, presented here, in which however much other information regarding plastic collapse may be obtained.

Even though the formulation via SGBEM, applied to plasticity problems, provides a high level of knowledge, computational difficulties have reduced the application of the method in practical engineering problems. Indeed, in literature there are very few applications, often utilizing simplified procedures to obtain the elastoplastic response.

In the present paper, a multidomain SGBEM strategy, based on an initial strain approach for two-dimensional body analysis in the hypothesis of elastic-perfectly plastic behaviour, von Mises materials, associated flow and plane strain condition, is shown.

Let us start from the discretization of the domain through a substructuring used following an SGBEM multidomain strategy $[11,12]$ for both the substructures (called macro-elements), generally of large dimensions, having merely elastic behaviour and the substructures (called bem-elements or bem-e), generally having very small dimensions, used in the elastoplastic phenomenon. Then, let us utilize the displacement method, introduced by Panzeca et al. [11], by imposing the regularity (or coupling) conditions at the interface boundaries among substructures, in which the unknowns are the interface nodal displacements. Subsequently, the self-equilibrium stress equation governing the elastoplastic problem and relating stresses, computed on each bem-e Gauss point, to plastic strains (treated as volumetric distortions) can be found. This equation contains an influence matrix (self-stress matrix) which is nonsymmetric, negative semi-definite and fully-populated. This matrix is used both to evaluate the predictors and to correct them by taking the stress back inside the elastic yield domain.

In the predictor phase, this equation permits one to locate all the active bem-elements, related to a load increment, that is, to define the active macro-zones whose bem-elements require a correction utilizing a path-following technique.

Then, in the corrector phase, the elastic solution is modified by a return mapping algorithm, based on the extremal path theory proposed by Ponter and Martin [13]. This algorithm was used for a simultaneous correction in all the active bem-elements that define the active macro-zones. The approximate solution of the previous nonlinear problem was obtained using the well-known Newton-Raphson (NR) method.

This approach shows a more rational and innovatory strategy regarding both the theoretical and computational aspects of the symmetric BEM. As a consequence of this research and of the approach presented here, some important characteristics are now indicated:

- The fundamental solutions are used in every substructure into which the body is subdivided, guaranteeing compatibility and equilibrium at any point in each substructure;

- The computation in a closed form of all the coefficients of the algebraic operators is carried out, and consequently it is possible to perform the analysis of substructures having any shape and dimension, without giving rise to numerical instability;

- It is possible to use the displacement method of the symmetric BEM, by imposing, at the interface of the substructures, the boundary conditions regarding compatibility and equilibrium in strong and weak form, respectively $[11,12]$;

- The transfer of the domain plastic strains into the boundary action is utilized [14, 15].

The main topics of the present paper regard:

- The updating of the plastic strains in the bem-elements, which define the active macro-zones in a nonlocal strategy, obtained through very low number of the plastic iterations, is performed; 
- The updating of the quantities governing the analysis process is performed at the end of each load increment, contrary to what happens in other formulations, which need continual correction of the solution for each plastic iteration;

- A substructuring of the domain where the plastic phenomenon takes place is made, leaving the remaining parts as macro-elements having elastic behaviour only, and involving a reduced number of boundary variables.

Finally, some examples developed using the Karnak.sGbem code [16] show the efficiency of the proposed approach.

\section{Main definitions:}

- $m$ substructures into which the domain is subdivided, distinguished into $r$ bem-elements having very small dimensions where the plastic strains may arise and in $(m-r)$ macro-elements having large dimensions and characterizing merely elastic behaviour;

- Active macro-zones: All the bem-elements which, at a generic load step, violate the plastic admissibility condition and therefore are subjected to a simultaneous corrector phase to define an admissible stress field;

\section{ELASTOPLASTIC ANALYSIS VIA MULTIDOMAIN SGBEM}

This section provides a detailed description of the procedure utilized to obtain the equations connecting all the quantities related to each bem-e involved in an elastoplastic analysis. These equations define the response, in terms of generalized tractions on the boundary and of stress inside the domain of the substructures (bem-elements), to the imposed plastic strains, within the SGBEM for multidomain type problems.

Let us consider the classical Somigliana Identities:

$$
\begin{aligned}
\mathbf{u} & =\int_{\Gamma} \mathbf{G}_{u u} \mathbf{f} \mathrm{d} \Gamma+\int_{\Gamma} \mathbf{G}_{u t}(-\mathbf{u}) \mathrm{d} \Gamma+\int_{\Omega} \mathbf{G}_{u u} \overline{\mathbf{b}} \mathrm{d} \Omega+\int_{\Omega} \mathbf{G}_{u \sigma} \boldsymbol{\epsilon}^{\mathrm{p}} \mathrm{d} \Omega \\
\mathbf{t} & =\int_{\Gamma} \mathbf{G}_{t u} \mathbf{f} \mathrm{d} \Gamma+\int_{\Gamma} \mathbf{G}_{t t}(-\mathbf{u}) \mathrm{d} \Gamma+\int_{\Omega} \mathbf{G}_{t u} \overline{\mathbf{b}} \mathrm{d} \Omega+\int_{\Omega} \mathbf{G}_{t \sigma} \boldsymbol{\epsilon}^{\mathrm{p}} \mathrm{d} \Omega \\
\boldsymbol{\sigma} & =\int_{\Gamma} \mathbf{G}_{\sigma u} \mathbf{f} \mathrm{d} \Gamma+\int_{\Gamma} \mathbf{G}_{\sigma t}(-\mathbf{u}) \mathrm{d} \Gamma+\int_{\Omega} \mathbf{G}_{\sigma u} \overline{\mathbf{b}} \mathrm{d} \Omega+\int_{\Omega} \mathbf{G}_{\sigma \sigma} \boldsymbol{\epsilon}^{\mathrm{p}} \mathrm{d} \Omega
\end{aligned}
$$

These provide the displacements, tractions and stresses in $\Omega$ caused by layered mechanical jumps f and double-layered kinematical ones $-\mathbf{u}$, both known and unknown boundary vectors, and by body forces $\overline{\mathbf{b}}$ and volumetric inelastic actions $\boldsymbol{\epsilon}^{\mathrm{p}}$ in the $\Omega$ domain. The operators $\mathbf{G}_{r s}$ are the fundamental solution matrices in which, on the basis of the symbology introduced by Maier and Polizzotto [1], the subscripts $r=u, t, \sigma$ and $s=u, t, \sigma$ indicate the effect ( $u$ displacement, $t$ traction, $\sigma$ stress in $x$ ) and the dual quantity in an energetic sense ( $u$ force, $t$ displacement jump, $\sigma$ volumetric distorsion in $x^{\prime}$ ) associated with the cause, respectively.

The vector $\epsilon^{\mathrm{p}}=\left[\varepsilon_{x}^{\mathrm{p}}, \varepsilon_{y}^{\mathrm{p}}, \varepsilon_{x y}^{\mathrm{p}}\right]^{\mathrm{T}}$ represents the plastic strain actions. Their presence requires domain integrals having singular kernels. These integrals may be considered as Cauchy Principal Values to which the Bui free term is associated. Otherwise, the employment of the regularization technique, applied for the purpose of cutting off the strong singularity, and of the subsequent Radial Integral Method (RIM) [17] permits us to transform the domain integrals into boundary ones for each bem-e. The reader can refer to Panzeca et al. [14] and Zito et al. [15] for a more detailed discussion of the computational aspects and the related implementation.

Let us consider a bidimensional body having domain $\Omega$ and boundary $\Gamma$, subjected to actions in its plane (Figure 1):

- Forces $\overline{\mathbf{f}}_{2}$ at the portion $\Gamma_{2}$ of free boundary,

- Displacements $\overline{\mathbf{u}}_{1}$ imposed at the portion $\Gamma_{1}$ of constrained boundary,

- Body forces $\overline{\mathbf{b}}$ and plastic strains $\epsilon^{\mathrm{p}}$ in $\Omega$. 


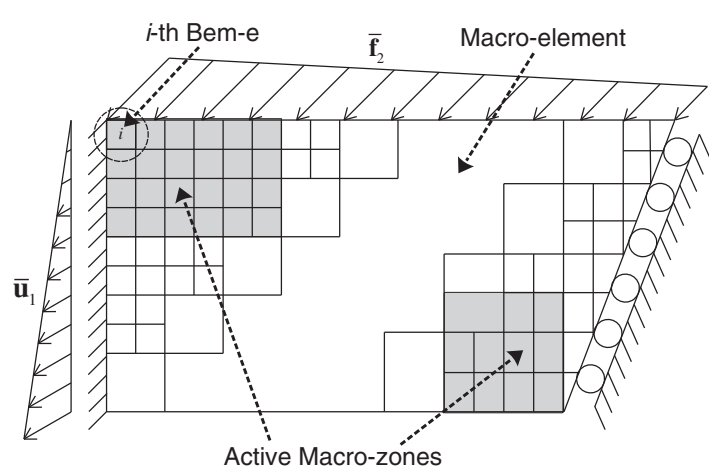

(a)

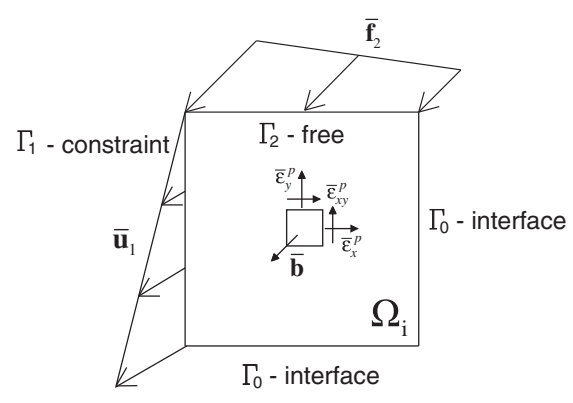

(b)

Figure 1. (a) The body subdivided into substructures, bem-elements and macro-zones, (b) the $i$-th bem-e subjected to boundary and domain actions.

Let us introduce the discretization of the domain into substructures, called bem-elements, where the plastic strain increments have to be evaluated in discrete terms and stored during the load-unload process and into macro-elements having elastic behaviour, only. For each substructure this subdivision involves the introduction of an interface boundary $\Gamma_{0}$ between contiguous substructures and, as a consequence, the birth of two new unknown quantities in the analysis problem, that is, the displacement $\mathbf{u}_{0}$ and the traction $\mathbf{t}_{0}$ vectors, both referred to interface boundaries.

We want to obtain the elastic response to the external actions in terms of displacements $\mathbf{u}_{2}$ on $\Gamma_{2}$, reactive forces $\mathbf{f}_{1}$ on $\Gamma_{1}$, but also in terms of displacements and tractions of the interface boundary $\Gamma_{0}$ of all the substructures and of the stresses $\sigma$ by using the symmetric boundary element displacement method [11,12]. Equations (1a) and (1b) are utilized to obtain, through the employment of their weighting according to the Galerkin theory, the algebraic operator coefficients necessary to perform the analysis phase; Equation (1c) is utilized to provide the stress field in the collocation points.

\subsection{Characteristic equations of the bem-e}

Let us consider any substructure in Figure 1(b), characterized by the boundary $\Gamma$ distinguished into free $\Gamma_{2}$, constrained $\Gamma_{1}$ and interface $\Gamma_{0}$ ones, generally not all present. For this substructure the following Dirichlet and Neumann conditions can be written:

$$
\mathbf{u}_{1}=\overline{\mathbf{u}}_{1} \quad \text { on } \Gamma_{1}, \quad \mathbf{t}_{2}=\overline{\mathbf{f}}_{2} \quad \text { on } \Gamma_{2} .
$$

If in these equations we introduce the Somigliana Identities of the displacements and tractions, the following boundary integral equations can be obtained:

$$
\begin{aligned}
& \int_{\Gamma_{1}} \mathbf{G}_{u u} \mathbf{f}_{1} \mathrm{~d} \Gamma_{1}+\int_{\Gamma_{2}} \mathbf{G}_{u t}\left(-\mathbf{u}_{2}\right) \mathrm{d} \Gamma_{2}+\int_{\Gamma_{0}} \mathbf{G}_{u u} \mathbf{f}_{0} \mathrm{~d} \Gamma_{0}+\int_{\Gamma_{0}} \mathbf{G}_{u t}\left(-\mathbf{u}_{0}\right) \mathrm{d} \Gamma_{0}+\int_{\Omega} \mathbf{G}_{u \sigma} \boldsymbol{\epsilon}^{\mathrm{p}} \mathrm{d} \Omega \\
& +\oint_{\Gamma_{1}} \mathbf{G}_{u t}\left(-\overline{\mathbf{u}}_{1}\right) \mathrm{d} \Gamma_{1}+\frac{1}{2} \overline{\mathbf{u}}_{1}+\int_{\Gamma_{2}} \mathbf{G}_{u u} \overline{\mathbf{f}}_{2} \mathrm{~d} \Gamma_{2}+\int_{\Omega} \mathbf{G}_{u u} \overline{\mathbf{b}} \mathrm{d} \Omega=\overline{\mathbf{u}}_{1} \\
& \int_{\Gamma_{1}} G_{t u} \mathbf{f}_{1} \mathrm{~d} \Gamma_{1}+\int_{\Gamma_{2}} G_{t t}\left(-\mathbf{u}_{2}\right) \mathrm{d} \Gamma_{2}+\int_{\Gamma_{0}} G_{t u} \mathbf{f}_{0} \mathrm{~d} \Gamma_{0}+\int_{\Gamma_{0}} G_{t t}\left(-\mathbf{u}_{0}\right) \mathrm{d} \Gamma_{0}+\int_{\Omega} \mathbf{G}_{t \sigma} \boldsymbol{\epsilon}^{p} \mathrm{~d} \Omega \\
& +\int_{\Gamma_{1}} G_{t t}\left(-\overline{\mathbf{u}}_{1}\right) \mathrm{d} \Gamma_{1}+\oint_{\Gamma_{2}} G_{t u} \overline{\mathbf{f}}_{2} \mathrm{~d} \Gamma_{2}+\frac{1}{2} \overline{\mathbf{f}}_{2}+\int_{\Omega} \mathbf{G}_{t u} \overline{\mathbf{b}} \mathrm{d} \Omega=\overline{\mathbf{f}}_{2}
\end{aligned}
$$

which written in symbolic form become

$$
\mathbf{u}_{1}\left[\mathbf{f}_{1},-\mathbf{u}_{2}, \mathbf{f}_{0},-\mathbf{u}_{0}, \boldsymbol{\epsilon}^{\mathrm{p}}\right]+\mathbf{u}_{1}\left[\overline{\mathbf{f}}_{2},-\overline{\mathbf{u}}_{1}^{C P V}, \overline{\mathbf{b}}\right]+\frac{1}{2} \overline{\mathbf{u}}_{1}=\overline{\mathbf{u}}_{1}
$$




$$
\mathbf{t}_{2}\left[\mathbf{f}_{1},-\mathbf{u}_{2}, \mathbf{f}_{0},-\mathbf{u}_{0}, \boldsymbol{\epsilon}^{\mathrm{p}}\right]+\mathbf{t}_{2}\left[\overline{\mathbf{f}}_{2}^{C P V},-\overline{\mathbf{u}}_{1}, \overline{\mathbf{b}}\right]+\frac{1}{2} \overline{\mathbf{f}}_{2}=\overline{\mathbf{f}}_{2},
$$

where the integrals with a circle in (3a) and (3b) and the apex $(C P V)$ in (3c) and (3d) define the presence of integrals as the Cauchy Principal Value, whereas the terms where 1/2 occurs are the free terms.

It is necessary to introduce the expressions of the displacements $\mathbf{u}_{0}$ and the tractions $\mathbf{t}_{0}$ related to the interface boundary $\Gamma_{0}$

$$
\begin{aligned}
\mathbf{u}_{0} & =\mathbf{u}_{0}\left[\mathbf{f}_{1},-\mathbf{u}_{2}, \mathbf{f}_{0},-\mathbf{u}_{0}^{C P V}, \epsilon^{\mathrm{p}}\right]+\frac{1}{2} \mathbf{u}_{0}+\mathbf{u}_{0}\left[\overline{\mathbf{f}}_{2},-\overline{\mathbf{u}}_{1}, \overline{\mathbf{b}}\right] \\
\mathbf{t}_{0} & =\mathbf{t}_{0}\left[\mathbf{f}_{1},-\mathbf{u}_{2}, \mathbf{f}_{0}^{C P V},-\mathbf{u}_{0}, \epsilon^{\mathrm{p}}\right]+\frac{1}{2} \mathbf{t}_{0}+\mathbf{t}_{0}\left[\overline{\mathbf{f}}_{2},-\overline{\mathbf{u}}_{1}, \overline{\mathbf{b}}\right]
\end{aligned}
$$

and to introduce the stress vector in the domain $\Omega$

$$
\sigma=\sigma\left[\mathbf{f}_{1},-\mathbf{u}_{2}, \mathbf{f}_{0},-\mathbf{u}_{0}, \boldsymbol{\epsilon}^{\mathrm{p}}\right]+\sigma\left[\overline{\mathbf{f}}_{2},-\overline{\mathbf{u}}_{1}, \overline{\mathbf{b}}\right] .
$$

Equations (3c), (3d) and (4a) can be rewritten in a different way, whereas Equations (4b) and (5) remain unchanged, thus obtaining

$$
\begin{gathered}
\mathbf{u}_{1}\left[\mathbf{f}_{1},-\mathbf{u}_{2}, \mathbf{f}_{0},-\mathbf{u}_{0}, \epsilon^{\mathrm{p}}\right]+\mathbf{u}_{1}\left[\overline{\mathbf{f}}_{2},-\overline{\mathbf{u}}_{1}^{C P V}, \overline{\mathbf{b}}\right]-\frac{1}{2} \overline{\mathbf{u}}_{1}=\mathbf{0} \\
\mathbf{t}_{2}\left[\mathbf{f}_{1},-\mathbf{u}_{2}, \mathbf{f}_{0},-\mathbf{u}_{0}, \boldsymbol{\epsilon}^{\mathrm{p}}\right]+\mathbf{t}_{2}\left[\overline{\mathbf{f}}_{2}^{C P V},-\overline{\mathbf{u}}_{1}, \overline{\mathbf{b}}\right]-\frac{1}{2} \overline{\mathbf{f}}_{2}=\mathbf{0} \\
\mathbf{u}_{0}\left[\mathbf{f}_{1},-\mathbf{u}_{2}, \mathbf{f}_{0},-\mathbf{u}_{0}^{C P V}, \boldsymbol{\epsilon}^{\mathrm{p}}\right]-\frac{1}{2} \mathbf{u}_{0}+\mathbf{u}_{0}\left[\overline{\mathbf{f}}_{2},-\overline{\mathbf{u}}_{1}, \overline{\mathbf{b}}\right]=\mathbf{0} \\
\mathbf{t}_{0}=\mathbf{t}_{0}\left[\mathbf{f}_{1},-\mathbf{u}_{2}, \mathbf{f}_{0}^{C P V},-\mathbf{u}_{0}, \boldsymbol{\epsilon}^{\mathrm{p}}\right]+\frac{1}{2} \mathbf{t}_{0}+\mathbf{t}_{0}\left[\overline{\mathbf{f}}_{2},-\overline{\mathbf{u}}_{1}, \overline{\mathbf{b}}\right] \\
\sigma=\sigma\left[\mathbf{f}_{1},-\mathbf{u}_{2}, \mathbf{f}_{0},-\mathbf{u}_{0}, \boldsymbol{\epsilon}^{\mathrm{p}}\right]+\sigma\left[\overline{\mathbf{f}}_{2},-\overline{\mathbf{u}}_{1}, \overline{\mathbf{b}}\right] .
\end{gathered}
$$

This simple rewriting of the Equations (3c), (3d) and (4a) requires a clearer explanation from a physical point of view. For this purpose, it is necessary to embed each substructure in an infinite domain having the same geometrical (thickness) and physical (Young's modulus and Poisson's ratio) characteristics as the substructure being examined. In this way, it is possible to characterize two boundaries, the boundaries $\Gamma$ of the body and $\Gamma^{+}$of the complementary domain. Equations (3c), (3d) and (4a) express the response on the boundary $\Gamma^{+}$of the complementary domain $\Omega_{\infty} \backslash \Omega$ and not on the actual boundary $\Gamma$, that is, $\mathbf{u}_{1}^{+}=\mathbf{0}$ on $\Gamma_{1}^{+}, \mathbf{t}_{2}^{+}=\mathbf{0}$ on $\Gamma_{2}^{+}, \mathbf{u}_{0}^{+}=\mathbf{0}$ on $\Gamma_{0}^{+}$. This evaluation modifies the sign of the free terms connected to the Cauchy Principal Value. In the fourth equation the traction $\mathbf{t}_{0}$ remains the same, that is, it will be evaluated on the actual boundary $\Gamma_{0}$.

More details are given in some papers by the same research group [11,12,18].

In the previous integral equations, $\epsilon^{\mathrm{p}}$ is the plastic strains vector, whose evaluation strategy is defined in the next section.

Let us introduce the boundary discretization into boundary elements by making the following modelling of all the known and unknown quantities:

$$
\mathbf{f}_{1}=\Psi_{\mathrm{f}} \mathbf{F}_{1}, \overline{\mathbf{f}}_{2}=\Psi_{\mathrm{f}} \overline{\mathbf{F}}_{2}, \mathbf{f}_{0}=\Psi_{\mathrm{f}} \mathbf{F}_{0}, \mathbf{u}_{2}=\Psi_{\mathrm{u}} \mathbf{U}_{2}, \overline{\mathbf{u}}_{1}=\Psi_{\mathrm{u}} \overline{\mathbf{U}}_{1}, \mathbf{u}_{0}=\Psi_{\mathrm{u}} \mathbf{U}_{0}, \boldsymbol{\varepsilon}^{\mathrm{p}}=\boldsymbol{\Psi}_{p} \mathbf{p}
$$

where $\boldsymbol{\Psi}_{\mathrm{f}}$ and $\boldsymbol{\Psi}_{\mathrm{u}}$ are appropriate shape functions regarding the boundary quantities, while $\boldsymbol{\Psi}_{\mathrm{p}}$ are domain shape functions used to model plastic strains $\mathbf{p}$ associated with the Gauss points of each bem-e and not the macro-elements with elastic behaviour. In this context the functions modelling the traction are assumed to have the same shape in all the boundary types, and hence also the 
functions modelling the displacements. Furthermore, the capital letters $\mathbf{F}$ and $\mathbf{U}$ indicate the nodal vectors of the forces $\left(\mathbf{F}_{1}\right.$ on $\Gamma_{1}$ and $\mathbf{F}_{0}$ on $\left.\Gamma_{0}\right)$ and of the displacements $\left(\mathbf{U}_{2}\right.$ on $\Gamma_{2}$ and $\mathbf{U}_{0}$ on $\left.\Gamma_{0}\right)$ defined on the boundaries.

In the examples treated in this paper, only one Gauss point is considered and the shape function modelling the plastic behaviour is assumed to be constant.

Let us now perform the weighting of Equations (6a)-(6d). For this purpose, the same shape functions as those modelling the causes have been employed, but introduced in an energetically dual way according to the Galerkin approach.

In this way it is possible to obtain the following block system:

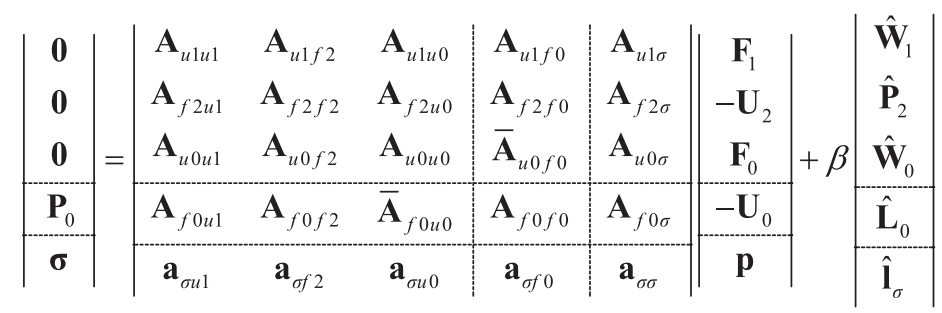

where the first and second rows represent the Dirichlet and Neumann conditions (6a,b) written in weighted form $\mathbf{W}_{1}^{+}=\int_{\Gamma_{1}} \boldsymbol{\psi}_{\mathrm{f}}^{\mathrm{T}}\left(\mathbf{u}_{1}-\overline{\mathbf{u}}_{1}\right)=\mathbf{0}$ and $\mathbf{P}_{2}^{+}=\int_{\Gamma_{2}} \boldsymbol{\psi}_{\mathrm{u}}^{\mathrm{T}}\left(\mathbf{t}_{2}-\overline{\mathbf{f}}_{2}\right)=\mathbf{0}$. The third $\left(\mathbf{W}_{0}^{+}=\int_{\Gamma_{1}} \boldsymbol{\psi}_{\mathrm{f}}^{\mathrm{T}} \mathbf{u}_{0}^{+}=\mathbf{0}\right)$ and the fourth row $\left(\mathbf{P}_{0}=\int_{\Gamma_{2}} \boldsymbol{\psi}_{\mathrm{u}}^{\mathrm{T}} \mathbf{t}_{0}\right)$ regard the weighting of the displacements and tractions at the interface boundary.

On the basis of the considerations regarding Equations (6a)-(6d), the four generalized vectors are referred to the boundaries $\Gamma_{1}^{+}, \Gamma_{2}^{+}, \Gamma_{0}^{+}$and $\Gamma_{0}$ previously introduced, but all evaluated in weighted form, that is, the weight of the response will be distributed by the shape functions introduced in accordance with the Galerkin theory. It is necessary to specify that in each boundary element two different shape functions, associated with the neighbouring nodes, have to be considered, whose sum must have unit value.

The influence matrix containing the first $4 \times 4$ block matrices is symmetric and moreover the term $\overline{\mathbf{A}}_{\mathrm{u} 0 \mathrm{f} 0}=\overline{\mathbf{A}}_{\mathrm{f0u} 0}^{\mathrm{T}}$ includes the weighting of both the $C P V$ integral and the free terms.

In Equation (8) the load vector containing both the body forces $\overline{\mathbf{b}}$ and the known boundary quantities $\left(-\overline{\mathbf{U}}_{1}, \overline{\mathbf{F}}_{2}\right)$ is amplified by $\beta$, which is the current estimate of the load multiplier in the updated load step.

The system introduced is similar to that found in $[11,12]$, where the characteristic equation is introduced to solve a multidomain problem by a method defined as the displacement method. Besides in the present formulation a column containing the weighted coefficients related to volumetric plastic strains $\mathbf{p}$ appears.

In contrast, the coefficients of the last row keep the stress meaning at the Gauss points, obtained modelling the causes and evaluating the field effect in accordance with the classical collocation approach.

To analyze the plastic phenomenon, the domain is discretized by introducing several bemelements in the zones where the plastic strains have to be computed. All the bem-elements have a boundary $\Gamma_{0}$, but only some bem-elements include the boundaries $\Gamma_{1}, \Gamma_{2}$, or both. As a consequence, in Equation (8) some rows and the corresponding columns have to be suppressed.

As can easily be observed, in Equation (8) the coefficients characterized by capital letters are computed as double integrals, whereas those characterized by small letters are coefficients obtained according to collocation BEM (Appendix A). Some of these integrals show singular or hypersingular kernels. These difficulties were overcome using different techniques: within the BEM we can mention the papers by Gray et al. [19] Portela and Aliabadi [20], Holzer [21], Guiggiani [22], and within the SGBEM, the papers by Frangi and Novati [23], Carini et al. [24], Panzeca et al. [18] and Terravecchia [25].

Specifically, the strategies developed by Panzeca et al. [18] and Terravecchia [25] permitted to develop a software called Karnak.sGbem [16], which is able to give solutions to plane structural 
systems, in-plane loaded, subjected to boundary and domain actions in the presence of both linear and nonlinear material behaviour and having any geometry.

Equation (8) can be expressed in a compact form in the following way:

$$
\begin{gathered}
\mathbf{0}=\mathbf{A} \mathbf{X}+\mathbf{A}_{0}\left(-\mathbf{U}_{0}\right)+\mathbf{A}_{\sigma} \mathbf{p}+\beta \hat{\mathbf{L}} \\
\mathbf{P}_{0}=\tilde{\mathbf{A}}_{0} \mathbf{X}+\mathbf{A}_{00}\left(-\mathbf{U}_{0}\right)+\mathbf{A}_{0 \sigma} \mathbf{p}+\beta \hat{\mathbf{L}}_{0} \\
\sigma=\mathbf{a}_{\sigma} \mathbf{X}+\mathbf{a}_{\sigma 0}\left(-\mathbf{U}_{0}\right)+\mathbf{a}_{\sigma \sigma} \mathbf{p}+\beta \hat{\mathbf{l}}_{\sigma},
\end{gathered}
$$

where the vector $\mathbf{X}$ collects the subvectors $\mathbf{F}_{1},\left(-\mathbf{U}_{2}\right)$ and $\mathbf{F}_{0}$, whereas the $\left(-\mathbf{U}_{0}\right)$ and $\mathbf{p}$ vectors characterize the nodal displacements of the interface zone, changed in sign, and the plastic strains at the Gauss points, respectively. The load vector $\hat{\mathbf{L}}$ collects the subvectors $\hat{\mathbf{W}}_{1}, \hat{\mathbf{W}}_{0}$ and $\hat{\mathbf{P}}_{2}$, all being the response in terms of weighted displacements on $\Gamma_{1}, \Gamma_{0}$ and weighted tractions on $\Gamma_{2}$ to both boundary and domain actions. The load vector $\hat{\mathbf{L}}_{0}$ is the response in terms of weighted tractions on $\Gamma_{0}$ to the boundary and domain actions.

The vector $\mathbf{P}_{0}$ represents the generalized (or weighted) traction vector defined in the boundary elements of the interface zone, obtained as a response to all the known and unknown actions, regarding boundary and domain quantities.

By performing a variable condensation through the replacement of the $\mathbf{X}$ vector extracted from Equation (9a) in Equations (9b) and (9c), one obtains

$$
\begin{gathered}
\mathbf{P}_{0}=\mathbf{D}_{00} \mathbf{U}_{0}+\mathbf{D}_{0 \sigma} \mathbf{p}+\beta \hat{\mathbf{P}}_{0} \\
\boldsymbol{\sigma}=\mathbf{d}_{\sigma 0} \mathbf{U}_{0}+\mathbf{d}_{\sigma \sigma} \mathbf{p}+\beta \hat{\boldsymbol{\sigma}},
\end{gathered}
$$

where

$$
\begin{gathered}
\mathbf{D}_{00}=\mathbf{A}_{0}^{T} \mathbf{A}^{-1} \mathbf{A}_{0}-\mathbf{A}_{00}, \mathbf{D}_{0 \sigma}=-\mathbf{A}_{0}^{T} \mathbf{A}^{-1} \mathbf{A}_{\sigma}+\mathbf{A}_{0 \sigma}, \hat{\mathbf{P}}_{0}=-\mathbf{A}_{0}^{T} \mathbf{A}^{-1} \hat{\mathbf{L}}+\hat{\mathbf{L}}_{0} \\
\mathbf{d}_{\sigma 0}=\mathbf{a}_{\sigma} \mathbf{A}^{-1} \mathbf{A}_{0}-\mathbf{a}_{\sigma 0}, \mathbf{d}_{\sigma \sigma}=-\mathbf{a}_{\sigma} \mathbf{A}^{-1} \mathbf{A}_{\sigma}+\mathbf{a}_{\sigma \sigma}, \hat{\boldsymbol{\sigma}}=-\mathbf{a}_{\sigma} \mathbf{A}^{-1} \hat{\mathbf{L}}+\hat{\mathbf{l}}_{\sigma}
\end{gathered}
$$

Equations (10a) and (10b) are two characteristic Equations written for each bem-e. They relate the generalized (or weighted) tractions $\mathbf{P}_{0}$ defined on the interface zone $\Gamma_{0}$ and the stresses $\sigma$ at Gauss points of the bem-e to the nodal displacements $\mathbf{U}_{0}$, the plastic strains $\mathbf{p}$ and the two load terms $\hat{\mathbf{P}}_{0}$ and $\hat{\boldsymbol{\sigma}}$ amplified by $\beta$, respectively. Moreover, $\mathbf{D}_{00}, \mathbf{D}_{0 \sigma}, \mathbf{d}_{\sigma 0}, \mathbf{d}_{\sigma \sigma}$ are the stiffness matrices, $\mathbf{D}_{00}$ and $\mathbf{d}_{\sigma \sigma}$ are square matrices, while $\mathbf{D}_{0 \sigma}$ and $\mathbf{d}_{\sigma 0}$ are rectangular ones.

\subsection{Assembled system}

Let us start by subdividing the body into $m$ substructures and considering Equations (10a) and (10b) for each of these. We thus obtain two global relations connecting all the generalized tractions and stresses related to the bem-elements considered, that is:

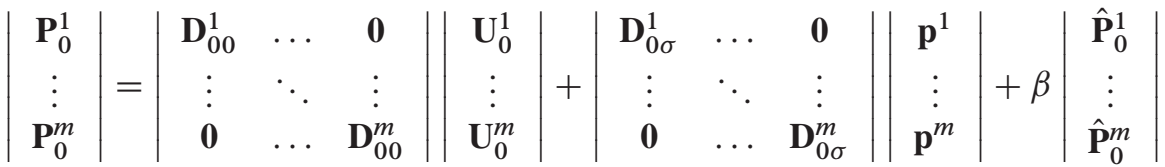

$$
\begin{aligned}
& \left|\begin{array}{c}
\sigma_{1} \\
\vdots \\
\boldsymbol{\sigma}_{r}
\end{array}\right|=\left|\begin{array}{ccc}
\mathbf{d}_{\sigma 0}^{1} & \ldots & \mathbf{0} \\
\vdots & \ddots & \vdots \\
\mathbf{0} & \ldots & \mathbf{d}_{\sigma 0}^{r}
\end{array}\right|\left|\begin{array}{c}
\mathbf{U}_{0}^{1} \\
\vdots \\
\mathbf{U}_{0}^{r}
\end{array}\right|+\left|\begin{array}{ccc}
\mathbf{d}_{\sigma \sigma}^{1} & \ldots & \mathbf{0} \\
\vdots & \ddots & \vdots \\
\mathbf{0} & \ldots & \mathbf{d}_{\sigma \sigma}^{r}
\end{array}\right|\left|\begin{array}{c}
\mathbf{p}^{1} \\
\vdots \\
\mathbf{p}^{r}
\end{array}\right|+\beta\left|\begin{array}{c}
\hat{\boldsymbol{\sigma}}^{1} \\
\vdots \\
\hat{\boldsymbol{\sigma}}^{r}
\end{array}\right|,
\end{aligned}
$$

where $r$ is the bem-e number of the discretized system and $(m-r)$ the macro-element number only having elastic behaviour. 
In compact form

$$
\begin{gathered}
\mathbf{P}_{0}=\mathbf{D}_{00} \mathbf{U}_{0}+\mathbf{D}_{0 \sigma} \mathbf{p}+\beta \hat{\mathbf{P}}_{0} \\
\boldsymbol{\sigma}=\mathbf{d}_{\sigma 0} \mathbf{U}_{0}+\mathbf{d}_{\sigma \sigma} \mathbf{p}+\beta \hat{\boldsymbol{\sigma}}
\end{gathered}
$$

formally equal to Equations (10a) and (10b), the first equation written for all the $m$ substructures (bem-elements and macro-elements), the second one written for the $r$ bem-elements, each having only one Gauss point.

All the substructures may take on any shape and dimension. No numerical instability is involved when the algebraic operators are employed in an analysis process, where bem-elements (small dimensions) and macro-elements (large dimensions) are used in the assembling phase. This happens because all the coefficients, hypersingular, singular and nonsingular, are evaluated in closed form, and utilized in the Karnak.sGbem program [16].

Let us introduce the compatibility among the nodal displacements of the adjacent substructures

$$
\mathbf{U}_{0}=\mathbf{H} \xi_{0},
$$

where $\mathbf{H}$ is the topological matrix

$$
\mathbf{H}=\left|\begin{array}{c}
\mathbf{H}^{1} \\
\vdots \\
\mathbf{H}^{m}
\end{array}\right|
$$

and $\xi_{0}$ the nodal displacement vector of the assembled system.

Let us introduce the equilibrium condition among generalized tractions at the interface boundaries

$$
\mathbf{H}^{\mathrm{T}} \mathbf{P}_{0}=\mathbf{0} .
$$

We obtain

$$
\begin{aligned}
& \mathbf{K}_{00} \xi_{0}+\mathbf{K}_{0 \sigma} \mathbf{p}+\beta \hat{\mathbf{f}}_{0}=\mathbf{0} \\
& \boldsymbol{\sigma}=\mathbf{k}_{\sigma 0} \boldsymbol{\xi}_{0}+\mathbf{k}_{\sigma \sigma} \mathbf{p}+\beta \hat{\boldsymbol{\sigma}},
\end{aligned}
$$

where

$$
\mathbf{K}_{00}=\mathbf{H}^{\mathrm{T}} \mathbf{D}_{00} \mathbf{H}, \mathbf{K}_{0 \sigma}=\mathbf{H}^{\mathrm{T}} \mathbf{D}_{0 \sigma}, \hat{\mathbf{f}}_{0}=\mathbf{H}^{\mathrm{T}} \hat{\mathbf{P}}_{0}, \mathbf{k}_{\sigma 0}=\mathbf{d}_{\sigma 0} \mathbf{H}, \mathbf{k}_{\sigma \sigma}=\mathbf{d}_{\sigma \sigma}
$$

$\mathbf{K}_{00}$ being symmetric, semi-definite negative and fully-populated.

By performing a new variable condensation through the replacement of the $\xi_{0}$ vector extracted from Equation (17a) into Equation (17b), we obtain

$$
\boldsymbol{\sigma}=\mathbf{Z} \mathbf{p}+\beta \hat{\boldsymbol{\sigma}}_{\mathrm{s}}
$$

where

$$
\mathbf{Z}=-\mathbf{k}_{\sigma 0} \mathbf{K}_{00}^{-1} \mathbf{K}_{0 \sigma}+\mathbf{k}_{\sigma \sigma}, \hat{\boldsymbol{\sigma}}_{\mathrm{s}}=-\mathbf{k}_{\sigma 0} \mathbf{K}_{00}^{-1} \hat{\mathbf{f}}_{0}+\hat{\boldsymbol{\sigma}} .
$$

Equation (19) provides the stress at the Gauss point of each bem-e depending on the volumetric plastic strains $\mathbf{p}$ and on the external actions $\hat{\boldsymbol{\sigma}}_{\mathrm{s}}$, the latter vector amplified by $\beta$. The first term of Equation (19) $\sigma^{\mathrm{p}}=\mathbf{Z} \mathbf{p}$ defines in all the discretized body $\Omega$ a self-equilibrium stress field having the same meaning of the classical definition $\mathbf{C}^{\mathrm{T}} \boldsymbol{\sigma}^{\mathrm{p}}=\mathbf{0}$ of the continuum, $\mathbf{C}^{\mathrm{T}}$ being the differential operator. This means that the term $\sigma^{\mathrm{p}}$ is in equibrium with zero body forces everywhere in $\Omega$ and with zero external forces on $\Gamma_{2}$.

The matrix $\mathbf{Z}$, defined as the self-stress influence matrix, is a square matrix having $3 r \times$ $3 r$ dimensions, with $r$ bem-elements number, fully-populated, nonsymmetric and semi-definite 
negative. The evaluation of this matrix only involves the elastic characteristic of the material and the structure geometry.

The matrix $\mathbf{Z}$ permits one to evaluate the elastic response in the Gauss point of each bem-e caused by the plastic strain vector $\mathbf{p}$, whereas the vector $\hat{\boldsymbol{\sigma}}_{s}$ collects the influence coefficients, as the response to the known external actions $\overline{\mathbf{F}}_{2},-\overline{\mathbf{U}}_{1}, \overline{\mathbf{b}}$.

Some remarks have to be made regarding the number of variables involved in performing the inversion of the matrices $\mathbf{A}$ in Equations (11a)-(11f) and $\mathbf{K}_{00}$ in Equations (20a) and (20b). Indeed, the dimension of matrix $\mathbf{A}$ depends on the restricted number of the nodes of the substructure being examined, whereas that of matrix $\mathbf{K}_{00}$ depends on the nodes of the assembled body. To limit the dimension of this latter matrix, it is possible to introduce in the discretization process several macro-elements having elastic behaviour only, thus reducing the number of the variables.

Other advantages are listed at the end of the introduction.

\section{THE ELASTOPLASTIC ALGORITHM}

The incremental elastoplastic analysis has to be performed through the employment of approaches involving plastically active bem-elements for each load increment, following an iterative process up to achievement of an established limit on the system's ductility. Two different alternatives will be shown, both using a return mapping algorithm through the NR procedure considering each plastically active bem-e singly or for entire macro-zones.

These approaches utilize Equation (19) to evaluate the trial stress in the predictor phase and the first term of Equation (19) to perform the corrector phase, to obtain the collapse load factor.

\subsection{Predictor phase}

Let us start computing the trial stresses, that is, the purely elastic response at the instant $n+1$ in each $r$ bem-elements of the discretized body.

For this purpose, Equation (19) provides all the predictors $\sigma_{(n+1)}^{*}$ as a function of the plastic strain $\mathbf{p}_{(n+1)}=\mathbf{p}_{(n)}+\Delta \mathbf{p}$, stored during the loading process, and of the load increments $\beta_{(n+1)}=\beta_{(n)}+\Delta \beta$, that is,

$$
\boldsymbol{\sigma}_{(n+1)}^{*}=\mathbf{Z} \mathbf{p}_{(n+1)}+\beta_{(n+1)} \hat{\boldsymbol{\sigma}}_{\mathrm{s}},
$$

where $\mathbf{Z}$ matrix is fully-populated and regards the $r$ bem-elements, obtained owing to the discretization.

It is necessary to perform a check on the plastic consistency condition at the Gauss points using the von Mises yield law, shown in Appendix B and here rewritten as follows:

$$
\boldsymbol{F}\left[\boldsymbol{\sigma}_{i(n+1)}^{*}\right]=\frac{1}{2} \boldsymbol{\sigma}_{i(n+1)}^{* \mathrm{~T}} \boldsymbol{M} \boldsymbol{\sigma}_{i(n+1)}^{*}-\sigma_{\mathrm{y}}^{2} \leqslant 0 \quad \text { with } i=1, \ldots, r
$$

$\sigma_{\mathrm{y}}$ being the yield stress and $\boldsymbol{M}$ the well-known matrix of constants.

In the $a$ bem-elements ( $a$ being active bem-elements number with $a \leqslant r$ ) where the latter inequality is violated, that is $\boldsymbol{F}\left[\sigma_{i(n+1)}^{*}\right]>0$, a corrector phase occurs, involving plastic strain increment computation.

In the following subsection a strategy to compute the plastic strains for each load step and at every bem-e is shown.

\subsection{Corrector phase}

Once the $a$ plastically active bem-elements are located, it is necessary to correct the stresses that are plastically not admissible by a return on the yield surface through the plastic strain increment computation using $\boldsymbol{\sigma}_{a}^{p}=\mathbf{Z}_{a a} \Delta \mathbf{p}_{a}$. 
To reach this goal two different strategies can be employed:

- The first one, called active macro-zone analysis, permits one to obtain the solution writing a single nonlinear equation system involving all the active bem-elements through simultaneous satisfaction of the plastic condition. This strategy shows the remarkable advantage of using a low plastic loop (or predictor-corrector loop) number and the drawback of using a Jacobian operator having large dimensions;

- The second one, called single active bem-e analysis, only considers one bem-e at a time and is characterized by very small Jacobian operators, but involves a high number of plastic loops.

3.2.1. Active macro-zone analysis. In a similar way to what it is shown in Appendix B to determine the elastoplastic return mapping algorithm in the continuum approach, the Equation (B.4a), written for a system discretized into $r$ bem-elements containing $a$ active ones, is again utilized. The aim is to perform the elastoplastic analysis at the $n+1$ load step, simultaneously in all the plastically active macro-zones identified in the previous predictor phase through the trial stress vector $\sigma_{a(n+1)}^{*}$, that is,

$$
\sigma_{a(n+1)}-\sigma_{a(n+1)}^{*}-\mathbf{Z}_{a a} \Delta \mathbf{p}_{a(n+1)}=\mathbf{0}
$$

where

$$
\boldsymbol{\sigma}_{a}=\left|\begin{array}{c}
\boldsymbol{\sigma}_{1} \\
\vdots \\
\boldsymbol{\sigma}_{a}
\end{array}\right|, \boldsymbol{\sigma}_{a}^{*}=\left|\begin{array}{c}
\boldsymbol{\sigma}_{1}^{*} \\
\vdots \\
\boldsymbol{\sigma}_{a}^{*}
\end{array}\right|, \Delta \mathbf{p}_{a}=\left|\begin{array}{c}
\Delta \mathbf{p}_{1} \\
\vdots \\
\Delta \mathbf{p}_{a}
\end{array}\right|, \mathbf{Z}_{a a}=\left|\begin{array}{ccc}
\mathbf{Z}_{11} & \cdots & \mathbf{Z}_{1 a} \\
\vdots & \ddots & \vdots \\
\mathbf{Z}_{a 1} & \cdots & \mathbf{Z}_{a a}
\end{array}\right|
$$

The $\mathbf{Z}_{a a}$ matrix coefficients are derived from the $\mathbf{Z}$ matrix present in Equation (19), by extracting the blocks relative to the $a$ plastically active bem-elements. The double index specifies the bem-elements where the stresses (effect) and the related plastic strains (cause) were considered.

Note that, comparing Equation (23) and Equation (B.4a), the correctors are opposite in sign. This is due to the event that the self-equilibrium stress matrix $\mathbf{Z}$ is negative semi-definite.

Thus, the return mapping analysis, shown in Appendix B for a continuum approach, is rewritten in terms of discrete variables and for all the active bem-elements, as follows:

$$
\begin{gathered}
\boldsymbol{\sigma}_{a}-\boldsymbol{\sigma}_{a}^{*}-\mathbf{Z}_{a a} \Delta \mathbf{p}_{a}=\mathbf{0} \\
\boldsymbol{F}\left[\boldsymbol{\sigma}_{a}\right] \leqslant \mathbf{0}, \Delta \boldsymbol{\Lambda}_{a} \geqslant \mathbf{0}, \Delta \boldsymbol{\Lambda}_{a} \boldsymbol{F}\left[\boldsymbol{\sigma}_{a}\right]=\mathbf{0},
\end{gathered}
$$

where the subscript $n+1$ has been omitted for simplicity.

In the hypothesis that, for each $k$-th active bem-e $(k=1, \ldots, a)$, the shape function defined in Equation $(7 \mathrm{~g})$ is the same as the shape function relating to the plastic multiplier, that is $\Delta \lambda_{k}=\psi_{\mathrm{p}} \Delta \Lambda_{k}$ with $\psi_{\mathrm{p}} \geqslant 0$, the plastic strain for the $k$-th active bem-e is expressed as

$$
\Delta \mathbf{p}_{k}=\Delta \Lambda_{k} \frac{\partial \boldsymbol{F}}{\partial \sigma_{k}}=\Delta \Lambda_{k} \boldsymbol{M} \boldsymbol{\sigma}_{k}
$$

The nonlinear system of Equations (B.5a) and (B.5b) is here written to be simultaneously solved in all the active bem-elements

$$
\left\{\begin{array}{l}
f_{\mathrm{I} h} \equiv \boldsymbol{\sigma}_{h}-\boldsymbol{\sigma}_{h}^{*}-\sum_{k=1}^{a} \Delta \Lambda_{k} \mathbf{Z}_{h k} \boldsymbol{M} \boldsymbol{\sigma}_{k}=\mathbf{0} \\
f_{\mathrm{II} h} \equiv \frac{1}{2} \boldsymbol{\sigma}_{h}^{\mathrm{T}} \boldsymbol{M} \boldsymbol{\sigma}_{h}-\sigma_{\mathrm{y}}^{2}=0 \quad \text { with } h=1, \ldots a
\end{array}\right.
$$

$\sigma_{h}$ being the total stress solution located on the yield surface of the elastic domain, $\sigma_{h}^{*}$ the trial stress, $\Delta \Lambda_{h} \mathbf{Z}_{h h} \boldsymbol{M} \boldsymbol{\sigma}_{h}$ the direct corrective component (local contribution) and $\sum_{k=1}^{a} \Delta \Lambda_{k} \mathbf{Z}_{h k} \boldsymbol{M} \boldsymbol{\sigma}_{k}$ with $k \neq h$ the indirect corrective components (nonlocal contributions), respectively. 
Equations (27a) and (27b) include a system of $4 \times a$ nonlinear equations in $4 \times a$ unknowns (three stress components $\sigma_{h}$ and the plastic multiplier $\Delta \Lambda_{h}$ for each active bem-e).

The approximate solution of this macro-zone nonlinear problem is here obtained by applying the NR procedure as follows:

$$
\left|\begin{array}{ccc:ccc}
\mathbf{I}-\Delta \Lambda_{1}^{j} \mathbf{Z}_{11} \boldsymbol{M} & \cdots & -\Delta \Lambda_{a}^{j} \mathbf{Z}_{1 a} \boldsymbol{M} & -\mathbf{Z}_{11} \boldsymbol{M} \boldsymbol{\sigma}_{1}^{j} & \cdots & -\mathbf{Z}_{1 a} \boldsymbol{M} \boldsymbol{\sigma}_{a}^{j} \\
\vdots & \ddots & \vdots & \vdots & \ddots & \vdots \\
-\Delta \Lambda_{a}^{j} \mathbf{Z}_{a 1} \boldsymbol{M} & \cdots & \mathbf{I}-\Delta \Lambda_{a}^{j} \mathbf{Z}_{a a} \boldsymbol{M} & -\mathbf{Z}_{a 1} \boldsymbol{M} \boldsymbol{\sigma}_{1}^{j} & \cdots & -\mathbf{Z}_{a a} \boldsymbol{M} \boldsymbol{\sigma}_{a}^{j} \\
\hdashline\left(\boldsymbol{\sigma}_{1}^{j}\right)^{T} \boldsymbol{M} & \cdots & \mathbf{0} & 0 & \cdots & 0 \\
\vdots & \ddots & \vdots & \vdots & \ddots & \vdots \\
\mathbf{0} & \cdots & \left(\boldsymbol{\sigma}_{a}^{j}\right)^{T} \boldsymbol{M} & 0 & \cdots & 0
\end{array}\right| \cdot\left|\begin{array}{c}
\boldsymbol{\sigma}_{1}^{j+1}-\boldsymbol{\sigma}_{1}^{j} \\
\vdots \\
\boldsymbol{\sigma}_{a}^{j+1}-\boldsymbol{\sigma}_{a}^{j} \\
\hdashline \Delta \Lambda_{1}^{j+1}-\Delta \Lambda_{1}^{j} \\
\vdots \\
\Delta \Lambda_{a}^{j+1}-\Delta \Lambda_{a}^{j}
\end{array}\right|=\left|\begin{array}{c}
\boldsymbol{f}_{I 1}^{j} \\
\vdots \\
-\boldsymbol{f}_{I a}^{j} \\
-f_{I I 1}^{j} \\
\vdots \\
-f_{I I a}^{j}
\end{array}\right|
$$

which written in a compact form becomes

$$
Y_{a}^{j+1}=Y_{a}^{j}-\mathbf{J}_{a a}^{-1}\left(\boldsymbol{Y}_{a}^{j}\right) \boldsymbol{f}\left(\boldsymbol{Y}_{a}^{j}\right)
$$

where the apex $j$ is related to the step-bystep procedure inside the $\beta_{(n+1)}$ load multiplier.

The vector $Y_{a}^{\mathrm{T}}=\left[\begin{array}{llllll}\sigma_{1}^{\mathrm{T}} & \ldots & \sigma_{a}^{\mathrm{T}}: \Delta \Lambda_{1} & \ldots & \Delta \Lambda_{a}\end{array}\right]$ characterizes the approximate solution in terms of stresses and plastic multipliers increments computed at the Gauss points of all the plastically active bem-elements. The Jacobian operator $\mathbf{J}_{a a}\left(\boldsymbol{Y}_{a}^{j}\right)$ usually has large dimension, and therefore the updating and inversion of this operator can prove onerous.

Using the self-stress $\mathbf{Z}$ matrix means that this procedure simultaneously provides the solution in all the plastically active bem-elements (active macro-zones). Furthermore, using this matrix gives rise to a nonlocal approach characterized by very low CPU times. Indeed, the plastic consistency condition proves to be satisfied in each bem-e simultaneously without the need for plastic iterations, thus giving a path-independent elastoplastic solution.

A restricted iterative process remains because through evaluation of the predictors it is necessary to verify the plastic consistency conditions of all the remaining bem-elements. In this phase the macro-zones may change, that is, some bem-elements drop out because they are purely elastic, whereas others come in because of their plastic behaviour. The effective end-step elastoplastic solution is reached when the yield condition is satisfied in all the bem-elements.

The existence of the solution of the nonlinear problem (28) is guaranteed, except when plastic collapse takes place.

3.2.2. Single active bem-e analysis. We will now show an alternative strategy making it possible to obtain the solution of the nonlinear problem for a single active bem-e. For this purpose, let us consider the $h$-th active bem-e. Equations (25a)-(25d) can be rewritten as follows:

$$
\begin{gathered}
\boldsymbol{\sigma}_{h}-\boldsymbol{\sigma}_{h}^{*}-\mathbf{Z}_{h h} \Delta \mathbf{p}_{h}=\mathbf{0} \\
F\left[\boldsymbol{\sigma}_{h}\right] \leqslant 0, \Delta \Lambda_{h} \geqslant 0, \Delta \Lambda_{h} F\left[\boldsymbol{\sigma}_{h}\right]=0 .
\end{gathered}
$$

The nonlinear system (27) is here rewritten for the single active bem-e

$$
\left\{\begin{array}{l}
\boldsymbol{f}_{\mathrm{I}} \equiv \boldsymbol{\sigma}_{h}-\boldsymbol{\sigma}_{h}^{*}-\Delta \Lambda_{h} \mathbf{Z}_{h h} \boldsymbol{M} \boldsymbol{\sigma}_{h}=\mathbf{0} \\
f_{\mathrm{II}} \equiv \frac{1}{2} \boldsymbol{\sigma}_{h}^{\mathrm{T}} \boldsymbol{M} \boldsymbol{\sigma}_{h}-\sigma_{\mathrm{y}}^{2}=0
\end{array},\right.
$$

where Equation (31a) only contains the local corrective contribution.

The approximate solution of this nonlinear problem is here obtained by applying the NR procedure as follows:

$$
\left|\begin{array}{c|c|c}
\mathbf{I}-\Delta \Lambda_{h}^{j} \mathbf{Z}_{h h} \boldsymbol{M} & -\mathbf{Z}_{h h} \boldsymbol{M} \boldsymbol{\sigma}_{h}^{j} \\
\hdashline\left(\boldsymbol{\sigma}_{h}^{j}\right)^{T} \boldsymbol{M} & 0 & 0 \\
\hdashline \Delta \Lambda_{h}^{j+1}-\Delta \Lambda_{h}^{j}
\end{array}\right|=\left|\begin{array}{c}
\boldsymbol{\sigma}_{h}^{j+1}-\boldsymbol{\sigma}_{h}^{j} \\
\hdashline-f_{I I}^{j}
\end{array}\right|
$$


which written in a compact form becomes

$$
\boldsymbol{Y}_{h}^{j+1}=\boldsymbol{Y}_{h}^{j}-\mathbf{J}_{h h}^{-1}\left(\boldsymbol{Y}_{h}^{j}\right) \boldsymbol{f}\left(Y_{h}^{j}\right) .
$$

The vector $\boldsymbol{Y}_{h}^{\mathrm{T}}=\left[\boldsymbol{\sigma}_{h}^{\mathrm{T}} ; \Delta \Lambda_{h}\right]$ is the approximate solution in terms of stresses and plastic multiplier computed at the Gauss point of the $h$-th active bem-e. Applying Equation (33) to all the active bem-elements, it is possible to obtain the solution of the elastoplastic problem by introducing a suitable number of plastic iterations. This strategy proves more onerous than macro-zone analysis, but it is characterized by the updating and inversion of a Jacobian operator $\mathbf{J}_{h h}\left(\boldsymbol{Y}_{h}\right)$ of small dimensions. In contrast, the solution given through Equation (33) is always guaranteed, in the plastic collapse phase too. This gives the method great robustness and reliability.

Finally, through the plastic strain vector $\mathbf{p}_{(n+1)}=\mathbf{p}_{(n)}+\Delta \mathbf{p}$, obtained using either the strategies shown in the Sections 3.2.1 or 3.2.2, and through the load multiplier $\beta_{(n+1)}=\beta_{(n)}+\Delta \beta$ the nodal quantities can easily be obtained as follows:

$$
\begin{aligned}
& \boldsymbol{\xi}_{0_{(n+1)}}=\mathbf{K}_{00}^{-1}\left(\mathbf{K}_{0 \sigma} \mathbf{p}_{(n+1)}+\beta_{(n+1)} \hat{\mathbf{f}}_{0}\right) \\
& \mathbf{X}_{(n+1)}=\mathbf{A}^{-1}\left(\mathbf{A}_{0} \mathbf{H} \boldsymbol{\xi}_{0_{(n+1)}}-\mathbf{A}_{\sigma} \mathbf{p}_{(n+1)}-\beta_{(n+1)} \hat{\mathbf{L}}\right)
\end{aligned}
$$

\section{THE ELASTOPLASTIC PROCEDURE}

In this section the sequence of steps concerning the proposed procedure for obtaining the numerical results is described.

Step 0: Load update $\beta_{(n+1)}=\beta_{(n)}+\Delta \beta$.

Step 1: Computing the elastic predictor $\boldsymbol{\sigma}_{(n+1)}^{*}=\mathbf{Z} \mathbf{p}_{(n+1)}+\beta_{(n+1)} \hat{\boldsymbol{\sigma}}_{\mathrm{s}}$.

Step 2: Check for yielding

If $F\left[\sigma_{(n+1)}^{*}\right] \leqslant T o l$, go to Step 0, or go to Step 7;

If $F\left[\sigma_{(n+1)}^{*}\right]>T o l$, go to Step 3 .

Step 3: Identification of the active bem-elements $\sigma_{a(n+1)}-\sigma_{a(n+1)}^{*}-\mathbf{Z}_{a a} \Delta \mathbf{p}_{a(n+1)}=\mathbf{0}(a$ being all the bem-elements of the active macro-zones) or $\sigma_{h(n+1)}-\sigma_{h(n+1)}^{*}-\mathbf{Z}_{h h} \Delta \mathbf{p}_{h(n+1)}=\mathbf{0}(h$ being the single active bem-e).

Step 4: Plastic corrector $\boldsymbol{Y}_{a}^{j+1}=\boldsymbol{Y}_{a}^{j}-\mathbf{J}_{a}^{-1}\left(\boldsymbol{Y}_{a}^{j}\right) \boldsymbol{f}\left(\boldsymbol{Y}_{a}^{j}\right)$ (active macro-zone analysis) or $\boldsymbol{Y}_{h}^{j+1}=\boldsymbol{Y}_{h}^{j}-\mathbf{J}_{h}^{-1}\left(\boldsymbol{Y}_{h}^{j}\right) \boldsymbol{f}\left(\boldsymbol{Y}_{h}^{j}\right)$ (single active bem-e analysis);

$j$ being the NR iteration number.

Step 5: Check for tolerance.

If $\boldsymbol{Y}^{j+1}-\boldsymbol{Y}^{j}>$ Tol go to Step 4

If $\boldsymbol{Y}^{j+1}-Y^{\boldsymbol{j}} \leqslant T o l$ go to Step 6

Step 6: Plastic strain vector update: $\mathbf{p}_{h(n+1)}=\mathbf{p}_{h(n)}+\Delta \Lambda_{h(n+1)} \boldsymbol{M} \boldsymbol{\sigma}_{h(n+1)}$, go to Step 1

Step 7: Nodal unknown update: $\left\{\begin{array}{l}\boldsymbol{\xi}_{0_{(n+1)}}=\mathbf{K}_{00}^{-1}\left(\mathbf{K}_{0 \sigma} \mathbf{p}_{(n+1)}+\beta_{(n+1)} \hat{\mathbf{f}}_{0}\right) \\ \mathbf{X}_{(n+1)}=\mathbf{A}^{-1}\left(\mathbf{A}_{0} \mathbf{H} \boldsymbol{\xi}_{0_{(n+1)}}-\mathbf{A}_{\sigma} \mathbf{p}_{(n+1)}-\beta_{(n+1)} \hat{\mathbf{L}}\right)\end{array} \quad\right.$ go to Step 0 or Exit.

It is important to note that the update of the nodal solution, given by the $\boldsymbol{\xi}_{0(n+1)}$ and $\boldsymbol{X}_{(n+1)}$ vectors, is performed at the end of the $n+1$ load increment, contrary to what happens in other formulations, which need continual correction of the nodal solution for each plastic iteration.

\section{NUMERICAL RESULTS}

To show the efficiency of the proposed method, three numerical tests were performed under the following hypotheses: plane strain condition, elastic-perfectly plastic von Mises law and associated plastic flow. 
These applications prove the performance of the eight-node linear bem-e, used in the zones where the plasticity is active. It allows one to obtain a perfectly plastic behaviour, consistently with the constitutive law adopted.

The advantage over other analysis methods lies in a better response, because it is based on satisfaction of the equilibrium and compatibility conditions inside each bem-e: this is due to the use of the fundamental solutions in the analysis process. Moreover, the present approach consists in using bem-e discretization of domain parts where the plastic phenomenon takes place, leaving the remaining parts as macro-elements having elastic behaviour only, and involving a reduced number of boundary variables.

In all the applications described, the S.I. unit is utilized, and therefore the stresses are defined in [MPa], and the geometry and displacements in [mm].

\subsection{Thin square plates with circular and square holes}

This section concerns two thin square plates with circular (Figure 2(a)) and square (Figure 2(b)) holes, subjected to a uniform tensile load $q$. Two different domain meshes were made using 128 and 252 bem-elements (Figure 2(c)), each having eight nodes, characterized by linear modelling of the boundary quantities. The material characteristics are the following: the Young's modulus $E=210,000 \mathrm{MPa}$ and the Poisson's ratio $v=0.3$, whereas the uniaxial yield value is $\sigma_{y}=200$ $\mathrm{MPa}$. Furthermore, the tolerance $\mathrm{Tol}=0.001$ was chosen in the return mapping analysis.

The aim is to compute the collapse load factor and to compare (see Table I) the numerical solutions, obtained through the strategy proposed in Section (3.2.1), with that obtained by other methods present in the literature [9,26-29].

In both cases (circular and square holes) shown in Figure 3 the behaviour proves to be similar. When a dense mesh (252 bem-elements) is introduced, the load-displacement curve shows that the plastic strains begin with gradualness, subsequently develop brusquely until collapse is reached. In the presence of a less dense mesh (128 bem-elements), the load-displacement curve shows that the beginning of plastic strains happens for a higher load value in comparison with the previous case but, because of greater stiffness assumed by the system during the plastic phase, the curve develops with continuity until collapse is reached when the load value is slightly higher than the value obtained in the previous case but with high inelastic displacements.

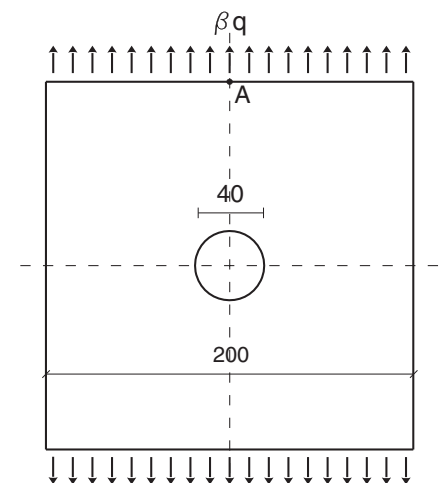

a)

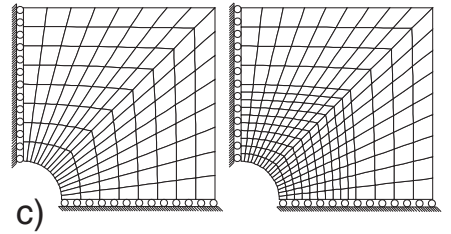

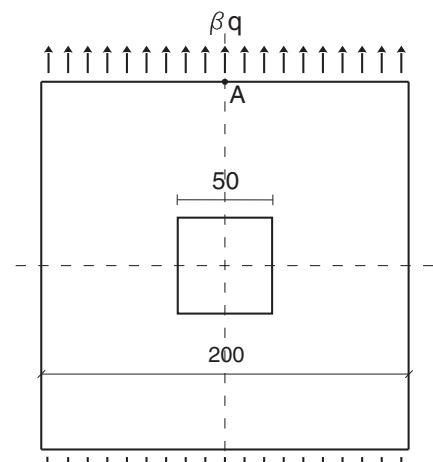

b)

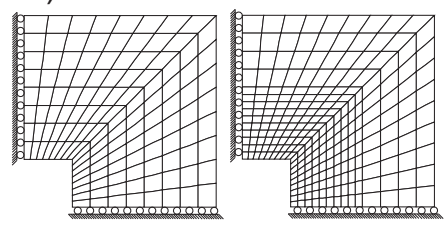

Figure 2. Thin square plates with circular and square holes: (a,b) geometric descriptions; (c) adopted meshes. 
Table I. Collapse load factor, obtained with active macro-zone SGBEM analysis compared with other formulations.

\begin{tabular}{llr}
\hline & \multicolumn{1}{c}{ Method } & $\beta \mathbf{q} / \sigma_{y}$ \\
\hline Circular hole & Exact (Gaydon and Mc Crum [26]) & 0.800 \\
& Lower bound (Borges et al. [27]) 423 elements & 0.800 \\
& SGBEM (Zhang et al. [9]) 128 cell elements & 0.801 \\
& Lower bound (Borges et al. [27]) 113 elements & 0.801 \\
& Casciaro and Cascini [28] & 0.803 \\
& SGBEM - Present approach 252 bem-elements & 0.805 \\
SGBEM - Present approach 128 bem-elements & 0.811 \\
& Belytschko and Hodge [29] & 0.693 \\
& SGBEM - Present approach 252 bem-elements & 0.733 \\
& SGBEM (Zhang et al. [9]) 128 cell elements & 0.747 \\
& SGBEM - Present approach 128 bem-elements & 0.755 \\
\hline
\end{tabular}

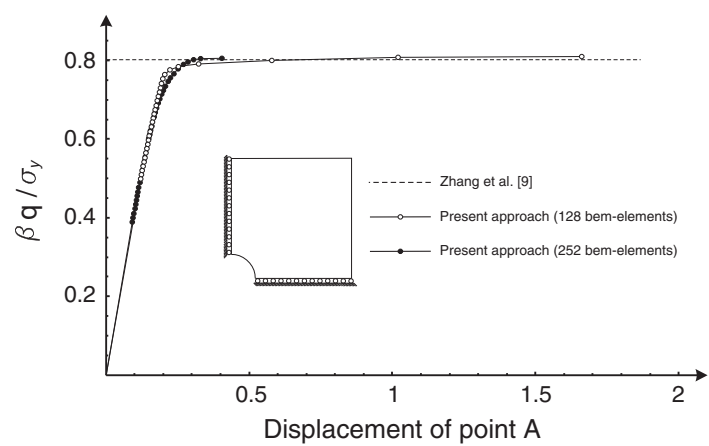

a)

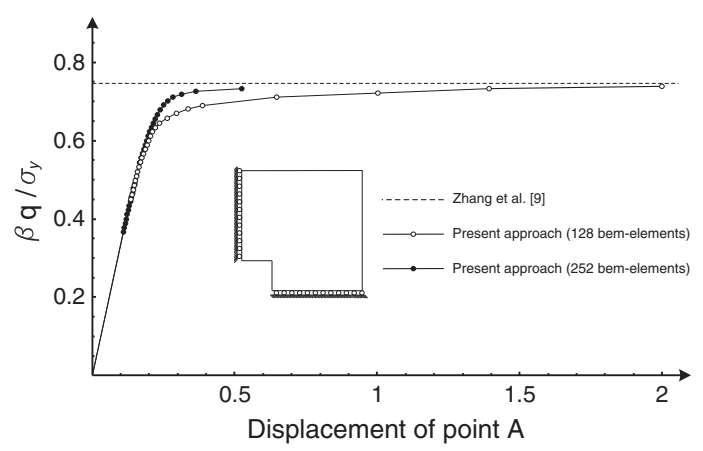

b)

Figure 3. Load-displacement curves.
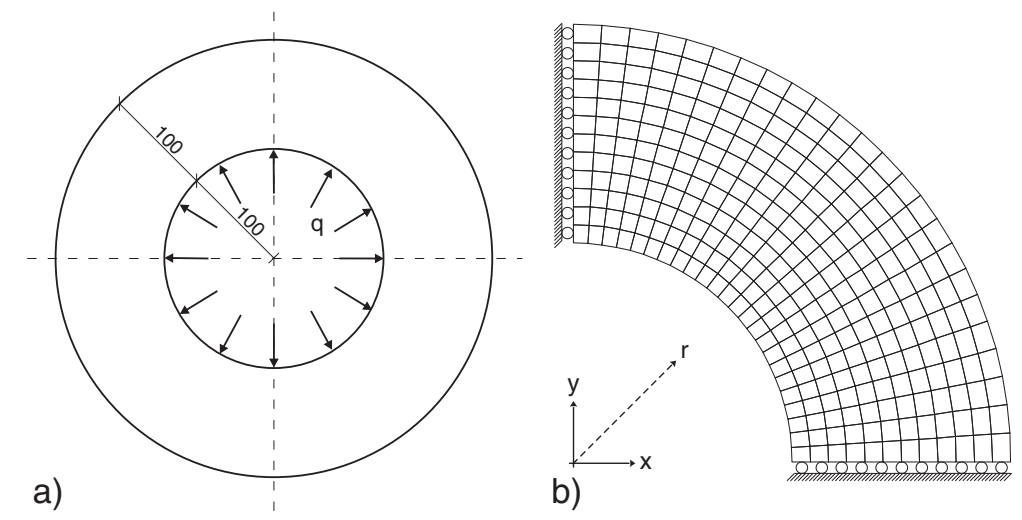

b)

Figure 4. Thick-wall cylinder under internal pressure: (a) geometric description and (b) adopted mesh.

In Figure 3 the curves regarding load multiplier $\beta$-displacement of point A defined in Figures 2(a) and (b) are shown and compared with the direct solution obtained by Zhang et al. [9] inside the SGBEM approach.

The strategy shown in Section (3.2.1) coupled with use of a bem-e having high performances permits us to obtain a collapse plastic multiplier with a low computational burden. Besides, when the update of plastic strains is quick, the load multiplier increment is reduced until the analysis involves numerical instability, thus characterizing the limit load.

It is to be noted that the solutions are in agreement with those obtained using other formulations. 


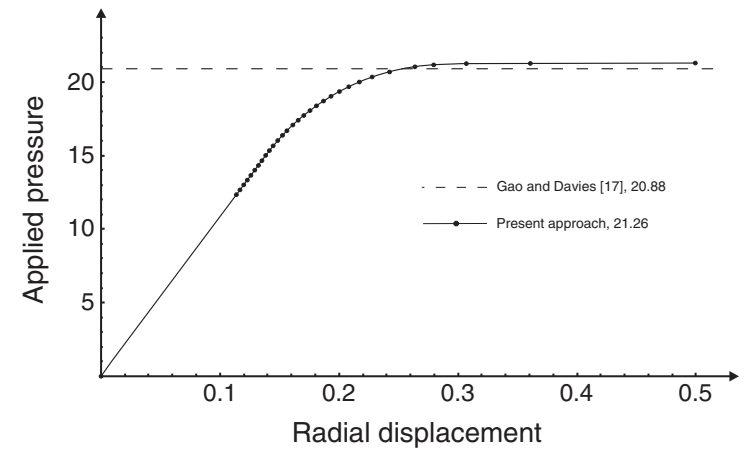

Figure 5. Pressure-radial displacement of inner face.

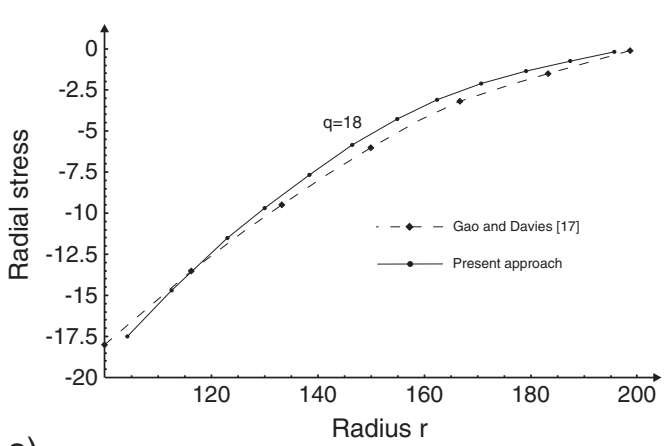

a)

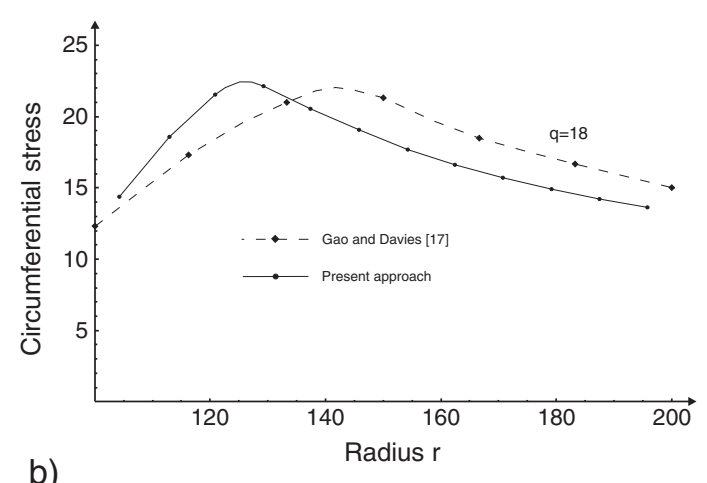

b)

Figure 6. Radial and circumferential stress distributions for pressure value $q=18 \mathrm{MPa}$.

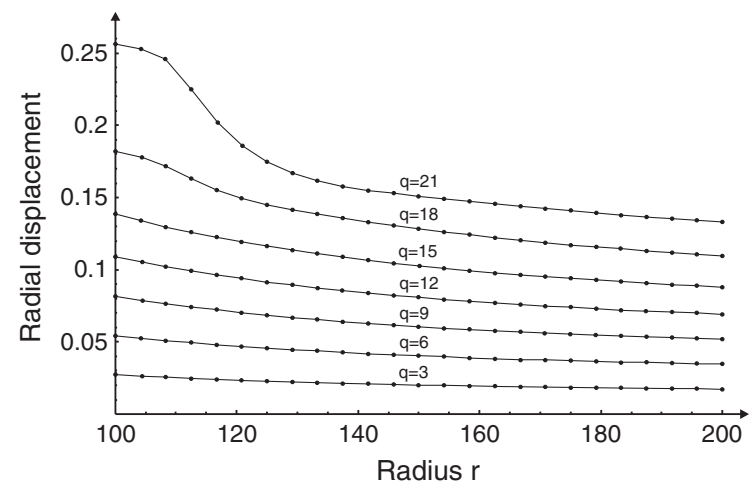

Figure 7. Radial displacements for different pressures.

\subsection{Thick-wall cylinder under internal pressure}

The second numerical example considered is a thick-wall cylinder subjected to internal pressure (Figure 4(a)), under plane strain conditions. The domain discretization consists of 288 bem-elements (Figure 4(b)), each having eight nodes and linear modelling of the boundary quantities. The material characteristics are the Young's modulus $E=21,000 \mathrm{MPa}$ and the Poisson's ratio $v=0.3$, whereas the uniaxial yield value $\sigma_{y}=30 \mathrm{MPa}$ and the initial pressure $q=1 \mathrm{MPa}$ are assumed. Furthermore, the same tolerance value $T o l=0.001$ was chosen as in the previous example.

The numerical solutions obtained, using the strategy proposed in Section 3.2.2, are compared with the results of Gao and Davies [17] in the sphere of a collocation approach. Figure 5 shows the pressure-radial displacement of the inner face. Plastic collapse occurs when the pressure reaches the value $q=21.26 \mathrm{MPa}$. 
The radial and the circumferential stress distributions for the pressure value $q=18 \mathrm{MPa}$ are plotted in Figures 6(a) and (b). Figure 7 shows the radial displacement for different pressure values $q=3,6, \ldots 21 \mathrm{MPa}$.

Some comparisons have to be made with the results of the analysis developed by Gao and Davies [17] inside the collocation BEM. The percentage difference of the limit load multiplier obtained in this paper in comparison with the value by Gao and Davies is about $2 \%$. In contrast, the percentage difference in valuating the radial and circumferential stresses is variable, reaching about $10 \%$. In detail, the radial stress obtained in this paper is bigger in comparison with that obtained by Gao and Davies and vice-versa for the circumferential stress, except in the intrados of the cylinder.

\subsection{Punch test on a T-section circular ring}

The third numerical example considered is a double punch test on a T-section ring, under plane strain conditions. The geometric characteristics are shown in Figure 8.

- The material characteristics of the ring are the Young's modulus $E=210,000 \mathrm{MPa}$ and the Poisson's ratio $v=0.29$, whereas the uniaxial yield value is $\sigma_{y}=200 \mathrm{MPa}$ and the initial unit load $q=1 \mathrm{MPa}$ is assumed.

- The material characteristics of the punch are $E=250,000 \mathrm{MPa}$ and $v=0.29$. In this case also, the tolerance value $T o l=0.001$ was considered.

Usually, to establish the active macro-zones where the plastic phenomenon occurs, an elastic analysis is performed beforehand.

Instead, in this application a preliminary elastoplastic analysis, using a trial mesh that considers the discretization of the whole domain into 250 four-node linear bem-elements (Figure 9(a)), was performed. This analysis, based on the strategy for single active bem-e (Section 3.2.2), defines where the active macro-zones are located. This made it possible to obtain an advantageous work mesh (Figure 9(b)) made up of 333 eight-node linear bem-elements, which characterize the plastic macro-zones and three macro-elements (two for the ring, flange and stem, and one for the punch), having elastic behaviour during all the load process.

The analysis performed with the macro-zone mesh shows that the plastic phenomenon only regards two macro-zones, inside the mesh adopted (Figure 9(b)).

The stress distribution is computed in the Gauss points of the bem-elements connected to the constraints.

In Figure 10 the load-displacement characteristic curves are shown, in both the trial and macrozone meshes, computing the displacement at the check point A at the top of the punch (Figure 8).

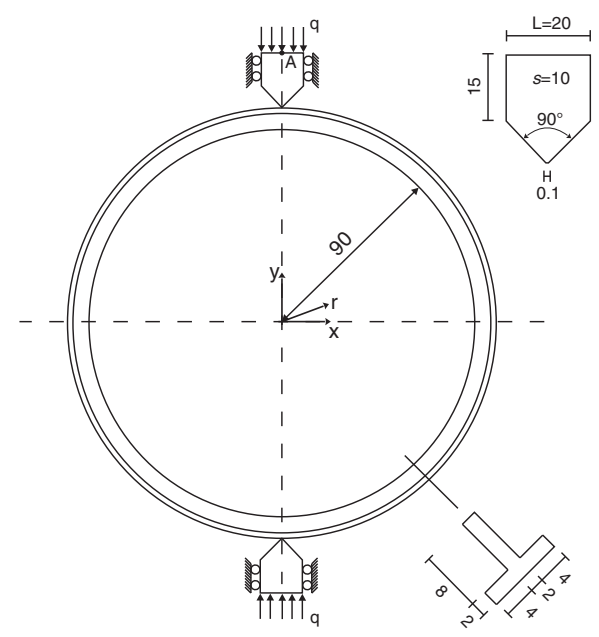

Figure 8. Punch test on a circular T-section ring: geometric description. 

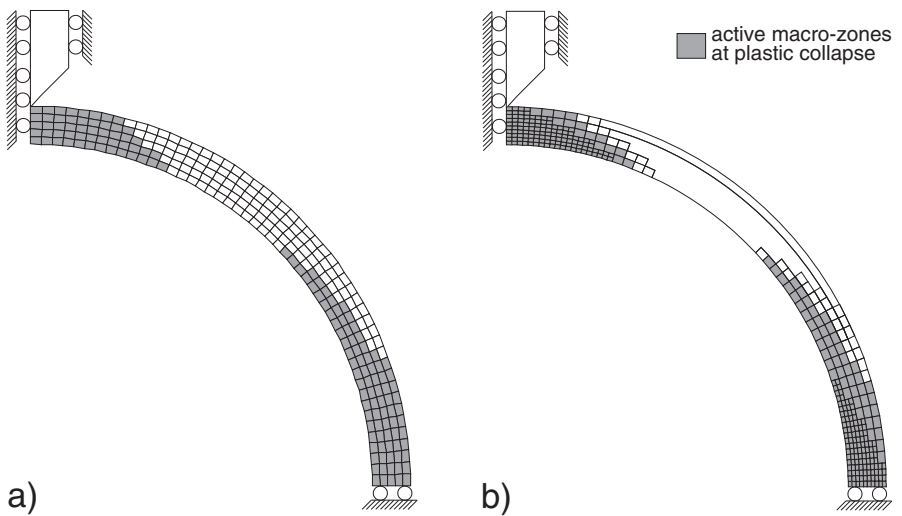

Figure 9. (a) Trial mesh and (b) macro-zone mesh.

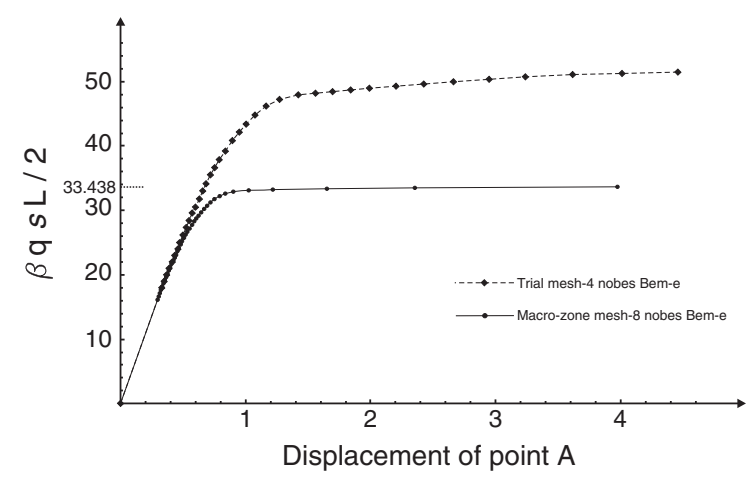

Figure 10. Load-displacement curves.

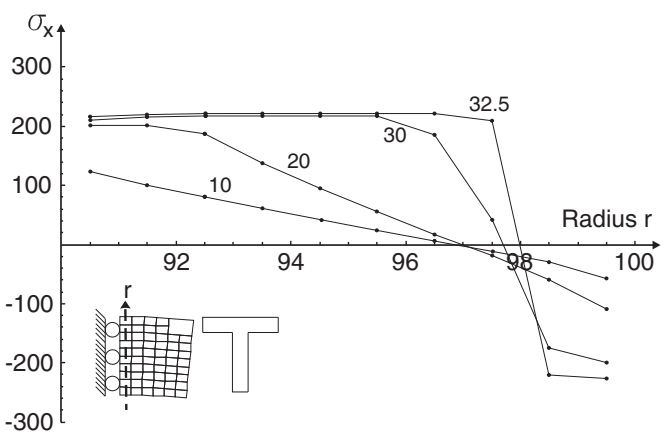

a)

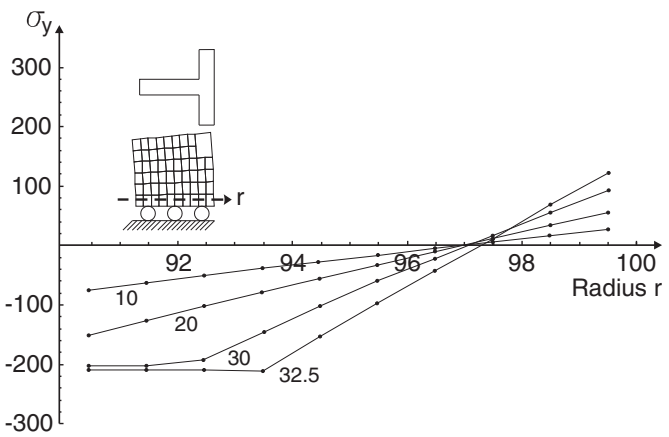

b)

Figure 11. Stress distributions for different load values: (a) $\sigma_{x}(r)$ in vertical section and (b) $\sigma_{y}(r)$ in horizontal section.

These curves show that the trial mesh (four-node bem-elements) fails in the evaluation of the plastic collapse multiplier because this test is characterized by excessively rigid behaviour of the structure.

In contrast, the macro-zone mesh (eight-node bem-elements) shows more efficient behaviour during plastic collapse, consistently with the constitutive law adopted, thus obtaining the plastic collapse multiplier equal to $35.438 \mathrm{daN}$.

Figure 11 shows the stress distributions in a vertical section $\sigma_{x}(r)$ (Figure 11(a)) and in a horizontal section $\sigma_{y}(r)$ (Figure 11(b)) for different load increments. In detail it shows the phenomenon of formation of the two plastic hinges that lead the structure to collapse. 


\section{CONCLUSIONS}

In this paper a strategy to perform incremental elastoplastic analysis using the SGBEM for multidomain type problems is shown. The use of a multidomain SGBEM strategy shows the advantage that one can easily consider step-wise phisically and geometrically not homogeneous materials. This strategy is accompanied by the transfer of all the domain integrals characterized by body forces and plastic strains into boundary integrals $[14,15]$ and the evaluation of all the coefficients in a closed form, relating both to boundary and domain actions.

Furthermore, using this strategy makes it possible to introduce a domain discretization exclusively in the zones of potential store of the plastic strains, leaving the rest of the structure as elastic macroelements, therefore governed by few boundary variables. This aspect makes the strategy proposed extremely advantageous.

This procedure provides a self-stress field governing the elastoplastic problem and connecting stresses, computed on each bem-e Gauss point, to plastic strains, treated as volumetric distortions, through an influence stiffness matrix (self-stress matrix) which is negative, semi-definite and fullypopulated. The same equation is used both to evaluate the predictors within the elastic phase and to correct them by taking the stress back inside the elastic yield domain.

In the first phase the use of a self-stress equation only offers the advantage of evaluating the predictors in a simple way. Indeed, this equation contains influence coefficients depending on both known imposed plastic strains and external actions amplified by the load multiplier. For the generic load increment, it permits one to locate all the bem-elements in which the plastic admissibility condition is violated, that is, to define the active macro-zones that require correction techniques. Then, in the second phase, the trial solution is corrected by a return mapping algorithm, which is defined according to the extremal path theory, used in this approach within a discrete problem.

The proposed algorithm permits the simultaneous correction of the elastic solution in all the plastically active bem-elements and utilizes the same self-stress equation in a nonlinear global system of $4 \times a$ equations in $4 \times a$ unknowns, where $a$ is the number of active bem-elements.

To prove the efficiency of the proposed strategy, some numerical tests, performed with a new procedure within the Karnak.sGbem code [16], are shown.

\section{APPENDIX A: WEIGHTED AND COLLOCATION COEFFICIENTS}

Coefficients defining the Equation (8) for the pseudostiffness operator:

$$
\begin{aligned}
& \mathbf{A}_{u 1 u 1}=\int_{\Gamma_{1}} \boldsymbol{\psi}_{f}^{\mathrm{T}} \int_{\Gamma_{1}} \mathbf{G}_{u u} \boldsymbol{\psi}_{f}=\mathbf{A}_{u 1 u 1}^{\mathrm{T}}, \mathbf{A}_{u 1 f 2}=\int_{\Gamma_{1}} \boldsymbol{\psi}_{f}^{\mathrm{T}} \int_{\Gamma_{2}} \mathbf{G}_{u t} \boldsymbol{\psi}_{u}=\mathbf{A}_{f 2, u 1}^{\mathrm{T}}, \mathbf{A}_{f 2 f 2}=\int_{\Gamma_{2}} \boldsymbol{\psi}_{u}^{\mathrm{T}} \int_{\Gamma_{2}} \mathbf{G}_{t t} \boldsymbol{\psi}_{u}=\mathbf{A}_{f 2 f 2}^{\mathrm{T}}, \\
& \mathbf{A}_{u 1 u 0}=\int_{\Gamma_{1}} \boldsymbol{\psi}_{f}^{\mathrm{T}} \int_{\Gamma_{0}} \mathbf{G}_{u u} \boldsymbol{\psi}_{f}=\mathbf{A}_{u 0 u 1}^{\mathrm{T}}, \mathbf{A}_{f 2 u 0}=\int_{\Gamma_{2}} \boldsymbol{\psi}_{u}^{\mathrm{T}} \int_{\Gamma_{0}} \mathbf{G}_{t u} \boldsymbol{\psi}_{f}=\mathbf{A}_{u 0 f 2}^{\mathrm{T}}, \mathbf{A}_{u 0 u 0}=\int_{\Gamma_{0}} \boldsymbol{\psi}_{f}^{\mathrm{T}} \int_{\Gamma_{0}} \mathbf{G}_{u u} \boldsymbol{\psi}_{f}=\mathbf{A}_{u 0 u 0}^{\mathrm{T}}, \\
& \mathbf{A}_{u 1 f 0}=\int_{\Gamma_{1}} \boldsymbol{\psi}_{f}^{\mathrm{T}} \int_{\Gamma_{0}} \mathbf{G}_{u t} \boldsymbol{\psi}_{u}=\mathbf{A}_{f 0 u 1}^{\mathrm{T}}, \mathbf{A}_{f 2 f 0}=\int_{\Gamma_{2}} \boldsymbol{\psi}_{u}^{\mathrm{T}} \int_{\Gamma_{0}} \mathbf{G}_{t t} \boldsymbol{\psi}_{u}=\int_{\Gamma_{0}} \boldsymbol{A}_{f}^{\mathrm{T}} \oint_{\Gamma_{0} f 2} \mathbf{G}_{u t} \boldsymbol{A}_{f 0 f 0}+\frac{1}{2} \int_{\Gamma_{0}} \boldsymbol{\psi}_{f}^{\mathrm{T}} \boldsymbol{\psi}_{u}=\overline{\mathbf{A}}_{f 0 u 0}^{\mathrm{T}} \int_{\Gamma_{0}} \mathbf{G}_{t t} \boldsymbol{\psi}_{u}=\mathbf{A}_{f 0 f 0}^{\mathrm{T}}, \\
& \mathbf{A}_{u 1 \sigma}=\int_{\Gamma_{1}} \boldsymbol{\psi}_{f}^{\mathrm{T}} \int_{\Omega} \mathbf{G}_{u \sigma} \boldsymbol{\psi}_{p}, \mathbf{A}_{f 2 \sigma}=\int_{\Gamma_{2}} \boldsymbol{\psi}_{u}^{\mathrm{T}} \int_{\Omega} \mathbf{G}_{t \sigma} \boldsymbol{\psi}_{p}, \\
& \mathbf{A}_{u 0 \sigma}=\int_{\Gamma_{0}} \boldsymbol{\psi}_{f}^{\mathrm{T}} \int_{\Omega} \mathbf{G}_{u \sigma} \boldsymbol{\psi}_{p}, \mathbf{A}_{f 0 \sigma}=\int_{\Gamma_{0}} \boldsymbol{\psi}_{u}^{\mathrm{T}} \int_{\Omega} \mathbf{G}_{t \sigma} \boldsymbol{\psi}_{p} \\
& \mathbf{a}_{\sigma u 1}=\int_{\Gamma_{1}} \mathbf{G}_{\sigma u} \boldsymbol{\psi}_{f}, \mathbf{a}_{\sigma f 2}=\int_{\Gamma_{2}} \mathbf{G}_{\sigma t} \boldsymbol{\psi}_{u}, \mathbf{a}_{\sigma u 0}=\int_{\Gamma_{0}} \mathbf{G}_{\sigma u} \boldsymbol{\psi}_{f}, \mathbf{a}_{\sigma f 0}=\int_{\Gamma_{0}} \mathbf{G}_{\sigma t} \boldsymbol{\psi}_{u} \\
& \mathbf{a}_{\sigma, \sigma}=\int_{\Omega} \mathbf{G}_{\sigma \sigma} \boldsymbol{\psi}_{p}
\end{aligned}
$$

and for the load vectors

$$
\hat{\mathbf{W}}_{1}=\int_{\Gamma_{1}} \boldsymbol{\psi}_{f}^{\mathrm{T}} \oint_{\Gamma_{1}} \mathbf{G}_{u t} \boldsymbol{\psi}_{u}\left(-\overline{\mathbf{U}}_{1}\right)+\frac{1}{2} \int_{\Gamma_{1}} \boldsymbol{\psi}_{f}^{\mathrm{T}} \boldsymbol{\psi}_{u}\left(-\overline{\mathbf{U}}_{1}\right)+\int_{\Gamma_{1}} \boldsymbol{\psi}_{f}^{\mathrm{T}} \int_{\Gamma_{2}} \mathbf{G}_{u u} \boldsymbol{\psi}_{f} \overline{\mathbf{F}}_{2}+\int_{\Gamma_{1}} \boldsymbol{\psi}_{f}^{\mathrm{T}} \int_{\Omega} \mathbf{G}_{u u} \overline{\mathbf{b}}
$$




$$
\begin{aligned}
& \hat{\mathbf{P}}_{2}=\int_{\Gamma_{2}} \boldsymbol{\psi}_{u}^{\mathrm{T}} \int_{\Gamma_{1}} \mathbf{G}_{t t} \boldsymbol{\psi}_{u}\left(-\overline{\mathbf{U}}_{1}\right)+\int_{\Gamma_{2}} \boldsymbol{\psi}_{u}^{\mathrm{T}} \oint_{\Gamma_{2}} \mathbf{G}_{t u} \boldsymbol{\psi}_{f} \overline{\mathbf{F}}_{2}-\frac{1}{2} \int_{\Gamma_{2}} \boldsymbol{\psi}_{u}^{\mathrm{T}} \boldsymbol{\psi}_{f} \overline{\mathbf{F}}_{2}+\int_{\Gamma_{2}} \boldsymbol{\psi}_{u}^{\mathrm{T}} \int_{\Omega} \mathbf{G}_{t u} \overline{\mathbf{b}} \\
& \hat{\mathbf{W}}_{0}=\int_{\Gamma_{0}} \boldsymbol{\psi}_{f}^{\mathrm{T}} \int_{\Gamma_{1}} \mathbf{G}_{u t} \boldsymbol{\psi}_{u}\left(-\overline{\mathbf{U}}_{1}\right)+\int_{\Gamma_{2}} \boldsymbol{\psi}_{f}^{\mathrm{T}} \int_{\Gamma_{2}} \mathbf{G}_{u u} \boldsymbol{\psi}_{f} \overline{\mathbf{F}}_{2}+\int_{\Gamma_{2}} \boldsymbol{\psi}_{f}^{\mathrm{T}} \int_{\Omega} \mathbf{G}_{u u} \overline{\mathbf{b}} \\
& \hat{\mathbf{L}}_{0}=\int_{\Gamma_{0}} \boldsymbol{\psi}_{u}^{\mathrm{T}} \int_{\Gamma_{1}} \mathbf{G}_{t t} \boldsymbol{\psi}_{u}\left(-\overline{\mathbf{U}}_{1}\right)+\int_{\Gamma_{0}} \boldsymbol{\psi}_{u}^{\mathrm{T}} \int_{\Gamma_{2}} \mathbf{G}_{t u} \boldsymbol{\psi}_{f} \overline{\mathbf{F}}_{2}+\int_{\Gamma_{0}} \boldsymbol{\psi}_{u}^{\mathrm{T}} \int_{\Omega} \mathbf{G}_{t u} \overline{\mathbf{b}} \\
& \hat{\mathbf{l}}_{\sigma}=\int_{\Gamma_{1}} \mathbf{G}_{\sigma t} \boldsymbol{\psi}_{u}\left(-\overline{\mathbf{U}}_{1}\right)+\int_{\Gamma_{2}} \mathbf{G}_{\sigma u} \boldsymbol{\psi}_{f} \overline{\mathbf{F}}+\int_{\Omega} \mathbf{G}_{\sigma u} \overline{\mathbf{b}} .
\end{aligned}
$$

\section{APPENDIX B: THE ELASTOPLASTIC RETURN MAPPING ALGORITHM}

In this Appendix the elastoplastic analysis is performed through a return mapping strategy.

Let us start from conditions defined in a continuum space through knowledge of stresses $\sigma_{(n)}$, total $\boldsymbol{\varepsilon}_{(n)}$ and plastic $\boldsymbol{\varepsilon}_{(n)}^{p}$ strains related at an instant $t_{(n)}$. The unknown response in terms of $\boldsymbol{\sigma}_{(n+1)}$, $\boldsymbol{\varepsilon}_{(n+1)}, \boldsymbol{\varepsilon}_{(n+1)}^{p}$ related to an instant $t_{(n+1)}$ has to be evaluated.

The integration of $\dot{\boldsymbol{\varepsilon}}^{p}$ in the interval $\left[t_{(n)}, t_{(n+1)}\right]$ leads to the following relation:

$$
\boldsymbol{\varepsilon}_{(n+1)}^{p}=\boldsymbol{\varepsilon}_{(n)}^{p}+\int_{t_{(n)}}^{t_{(n+1)}} \dot{\lambda} \frac{\partial F}{\partial \boldsymbol{\sigma}} \mathrm{d} t
$$

where $F$ is the function defining the elastic domain and $\dot{\lambda}$ is the plastic multiplier increment.

According to the theory proposed by Ponter and Martin [13], let us assume that the loading process be defined by an extremal path [30,31], the latter being an elastic path connecting, in the stress space, the starting point $\sigma_{(n)}$ and the final point $\sigma_{(n+1)}$, thus assuming that the plastic process takes place in the final point. Thus the previous equation takes the form:

$$
\boldsymbol{\varepsilon}_{(n+1)}^{p}=\boldsymbol{\varepsilon}_{(n)}^{p}+\left.\Delta \lambda_{(n+1)} \frac{\partial F}{\partial \boldsymbol{\sigma}}\right|_{n+1} .
$$

As a consequence, under the extremal path hypothesis the plastic response is path-independent.

The elastic predictor $\sigma_{(n+1)}^{*}$ is a function of the initial conditions $\sigma_{(n)}$ and of the load increment, that is,

$$
\sigma_{(n+1)}^{*}=\sigma_{(n)}+\mathbf{E} \Delta \varepsilon[\Delta \mathbf{u}]
$$

E being the elasticity tensor.

Then let us introduce the classic equations governing the elastoplasticity

$$
\begin{gathered}
\boldsymbol{\sigma}_{(n+1)}-\boldsymbol{\sigma}_{(n+1)}^{*}+\mathbf{E} \Delta \boldsymbol{\varepsilon}_{(n+1)}^{p}=\mathbf{0} \\
F\left[\boldsymbol{\sigma}_{(n+1)}\right] \leqslant 0, \Delta \lambda_{(n+1)} \geqslant 0, \Delta \lambda_{(n+1)} F\left[\boldsymbol{\sigma}_{(n+1)}\right]=0
\end{gathered}
$$

If $F\left[\sigma_{(n+1)}^{*}\right]>0$, the stress has to be corrected and a return mapping algorithm is required to evaluate the elastoplastic response. Equations (B.4a)-(B.4d) are solved by enforcing, as an equality, the yield condition, that is,

$$
\left\{\begin{array}{l}
\sigma_{(n+1)}-\sigma_{(n+1)}^{*}+\left.\Delta \lambda_{(n+1)} \mathbf{E} \frac{\partial F}{\partial \boldsymbol{\sigma}}\right|_{n+1}=\mathbf{0} \\
F\left[\sigma_{(n+1)}\right]=0
\end{array} .\right.
$$

The latter nonlinear equations system has three stress components $\sigma_{(n+1)}$ and the plastic multiplier increment $\Delta \lambda_{(n+1)}$ as unknowns, $\sigma_{(n+1)}^{*}$ being the elastic predictor and $\Delta \lambda_{(n+1)} \mathbf{E} \partial F / \partial \boldsymbol{\sigma}_{(n+1)}$ the plastic corrector, respectively. 
In particular, with regard to the case of a two-dimensional continuous solid and in the hypothesis of a plane strain condition, we utilize the elastic-perfectly plastic von Mises law

$$
F\left[\sigma_{(n+1)}\right]=\frac{1}{2} \sigma_{(n+1)}^{T} M \sigma_{(n+1)}-\sigma_{\mathrm{y}}^{2}
$$

$\sigma_{\mathrm{y}}$ being the yield stress and $M$ a matrix of constants [31].

As a consequence Equations (B.5) take the form

$$
\left\{\begin{array}{l}
\mathbf{f}_{\mathrm{I}} \equiv \sigma_{(n+1)}-\boldsymbol{\sigma}_{(n+1)}^{*}+\Delta \lambda{ }_{(n+1)} \mathbf{E} \boldsymbol{M} \boldsymbol{\sigma}_{(n+1)}=\mathbf{0} \\
f_{\mathrm{II}} \equiv \frac{1}{2} \sigma_{(n+1)}^{T} \boldsymbol{M} \boldsymbol{\sigma}_{(n+1)}-\sigma_{\mathrm{y}}^{2}=0
\end{array},\right.
$$

where the unknowns $\sigma_{(n+1)}$ and $\Delta \lambda_{(n+1)}$ of this nonlinear problem can be obtained by applying the NR procedures.

\section{REFERENCES}

1. Maier G, Polizzotto C. A Galerkin approach to boundary element elastoplastic analysis. Computer Methods in Applied Mechanics and Engineering 1987; 60:175-194.

2. Polizzotto C. An energy approach to the boundary element method. Part I: Elastic solids. Computer Methods in Applied Mechanics and Engineering 1988; 69:167-184.

3. Polizzotto C. An energy approach to the boundary element method. Part II: Elastic-plastic solids. Computer Methods in Applied Mechanics and Engineering 1988; 69:263-276.

4. Maier G, Miccoli S, Novati G, Perego U. Symmetric Galerkin boundary element method in plasticity and gradient plasticity. Computational Mechanics 1995; 17:115-129.

5. Frangi A, Maier G. A Dynamic elastic-plastic analysis by a symmetric Galerkin boundary element method with time-indipendent kernels. Computer Methods in Applied Mechanics and Engineering 1999; 171:281-308.

6. Polizzotto C, Zito M. BIEM-based variational principles for elastoplasticity with unilateral contact boundary conditions. Engineering Analysis with Boundary Elements 1998; 21:329-338.

7. Liu Q, Cen Z, Xu B. Plastic analysis by quasi-high order Galerkin BEM. Engineering Structures 1997; 19:1050-1056.

8. Panzeca T. Shakedown and limit analysis by the boundary integral equation method. European Journal of Mechanics A/Solids 1992; 11:685-699.

9. Zhang X, Liu Y, Zhao Y, Cen Z. Lower bound limit analysis by the symmetric Galerkin boundary element method and Complex method. Computer Methods in Applied Mechanics and Engineering 2002; 191:1967-1982.

10. Zhang X, Liu Y, Cen Z. Boundary element methods for lower bound limit and shakedown analysis. Engineering Analysis with Boundary Elements 2004; 28:905-917.

11. Panzeca T, Cucco F, Terravecchia S. Symmetric Boundary Element Method versus Finite Element Method. Computer Methods in Applied Mechanics and Engineering 2002; 191:3347-3367.

12. Panzeca T, Salerno M, Terravecchia S. Domain decomposition in the symmetric boundary element method analysis. Computational Mechanics 2002; 28:191-201.

13. Ponter ARS, Martin JB. Some extremal properties and energy theorems for inelastic materials and their relationship to the deformation theory of plasticity. Journal of the Mechanics and Physics of Solids 1972; 20:281-300.

14. Panzeca T, Terravecchia S, Zito L. Computational aspects in 2D SBEM analysis with domain inelastic actions. International Journal for Numerical Methods in Engineering 2010; 82:184-204.

15. Zito L, Parlavecchio E, Panzeca T. On the computational aspects of a symmetric multidomain BEM approach for elastoplastic analysis. Journal of Strain Analysis for Engineering Design 2011; 46:103-120.

16. Cucco F, Panzeca T, Terravecchia S. The program Karnak.sGbem Release 2.1, Palermo University, 2002.

17. Gao XW, Davies TG. An effective boundary element algorithm for $2 \mathrm{D}$ and $3 \mathrm{D}$ elastoplastic problem. International Journal of Solids and Structures 2000; 37:4987-5008.

18. Panzeca T, Fujita YH, Salerno M. Direct stiffness matrices of BEs in the Galerkin BEM formulation. European Journal of Mechanics A/Solids 2001; 20:277-298.

19. Gray LJ, Martha LF, Ingraffea AR. Hypersingular integrals in boundary element fracture analysis. International Journal for Numerical Methods in Engineering 1990; 29:1135-1158.

20. Portela A, Aliabadi MH. Dual Boundary Element Method: Effective implementation for crack problem. International Journal for Numerical Methods in Engineering 1992; 33:1269-1287.

21. Holzer S. How to deal with hypersingular integrals in the symmetric BEM. Communication in Numerical Methods in Engineeering 1993; 9:219-232.

22. Guiggiani M. Hypersingular boundary integral equation have an additional free term. Computational Mechanics 1995; 16:245-248.

23. Frangi A, Novati G. Symmetric BE method in two-dimensional elasticity: evaluation of double integrals for curved elements. Computational Mechanics 1996; 19:58-68. 
24. Carini A, Diligenti M, Maranesi P, Zanella M. Analytical integrations for two-dimensional elastic analysis by the symmetric Galerkin boundary element method. Computational Mechanics 1999; 23:308-323.

25. Terravecchia S. Closed form coefficients in the symmetric boundary element approach. Engineering Analysis with Boundary Elements 2006; 30:479-488.

26. Gaydon FA, Mc Crum AW. A theoretical investigation of the yield point loading of a square plate with a central circular hole. Journal of the Mechanics and Physics of Solids 1954; 2:156-169.

27. Borges LA, Zouain N, Huespe AE. A nonlinear optimization procedure for limit analysis. European Journal of Mechanics A/Solids 1996; 15:487-512.

28. Casciaro R, Cascini L. A mixed formulation and mixed finite elements for limit analysis. Intenational Journal for Numerical Methods in Engineering 1982; 18:211-243.

29. Belytschko T, Hodge PG. Plane stress limit analysis by finite element. Journal of Engineering Mechanics ASCE 1970; 96:931-944.

30. Ortiz M, Martin JB. Symmetry-preserving return mapping algorthms and incrementally extremal paths: a unification of concepts. International Journal for Numerical Methods in Engineering 1989; 28:1839-1853.

31. Bilotta A, Casciaro R. A high-performance element for the analysis of 2D elastoplastic continua. Computer Methods in Applied Mechanics and Engineering 2007; 196:818-828. 